\title{
THE RITUAL
}

OF

\section{THE METHODIST CHURCH,}

WITH

FORMS OF PRAYER.

TRANSLATED INTO CREE, AND WRITTEN IN SYLLABICS,

BY

REV. J. MCDOUGALL

AND

REV. E. B. GLASS, B.A.

PM989

M42 

OF

SOA

THE METHODIST/CHURCH,

WITH

FORMS OF PRAYER.

TRANSLATED INTO CREE, AND WRITTEN IN SYLLABICS,

BY

REV. J. MCDOUGALL

AND

REV. E. B. GLASS, B.A.

TORONTO :

METHODIST MISSIONARY SOCIETY. 
prips 99

$M+2$

$c p^{2}$ 


\section{THE RITUAL}

OF

\section{THE METHODIST CHURCH.}

\section{BAPTISM OF INFANTS.}

$\Delta ৬ \Gamma_{11} \nabla \Delta \cdot \Delta r \| \dot{C} \cdot \Delta \cdot a$.

r $b_{11} \dot{1}_{11}(9 \Delta \cdot)$.

1. $\nabla r b_{11} \dot{\Delta}_{11} \dot{C}_{11} r, \Delta \dot{j \cdot r} h$.

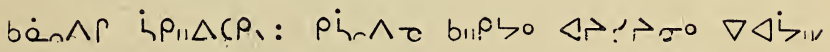

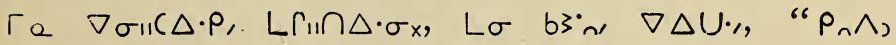

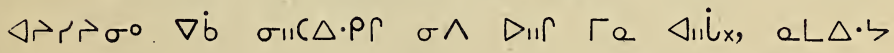

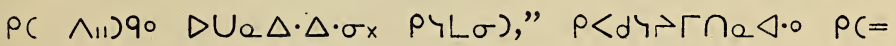

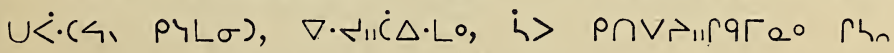

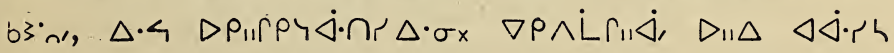

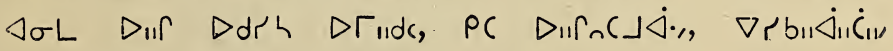

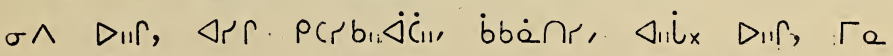
$p\left(\wedge_{11}\right) b \cdot \| \Delta_{11}$ ל $\left.3^{\circ}, \quad \nabla b \dot{Q} \Gamma \Delta \zeta \Gamma_{11} \nabla \Delta \cdot d \Gamma d x, \quad \nabla d r \quad \Gamma\right)_{\sigma}$

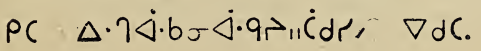


4

$\left(\nabla d r \quad \Delta b \Gamma_{\|} \nabla \Delta \cdot r \sigma^{\circ} p\left(\Delta U \cdot 0,-\Delta b \Gamma_{11} \Delta \dot{\zeta} \dot{C}_{0}\right)\right.$

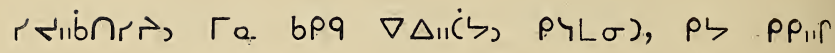

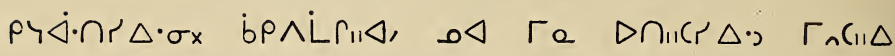

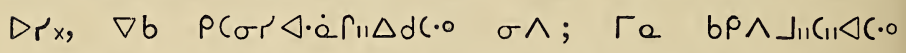

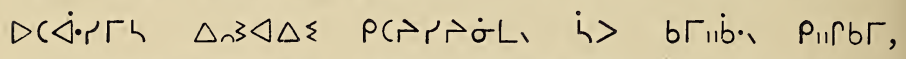

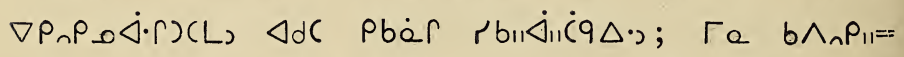
Cn(b) $\sigma \wedge$ DL Dir bér $\Delta r \| l \cdot \Delta \cdot)$; 「e $\nabla d c$ b $\Delta=$

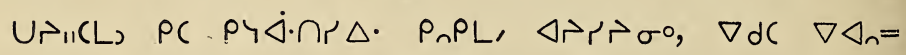

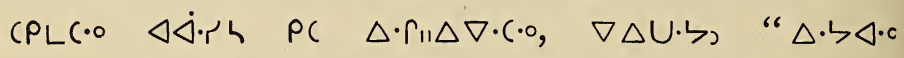

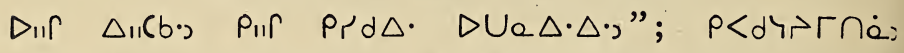

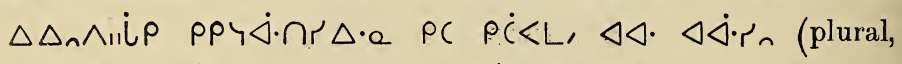

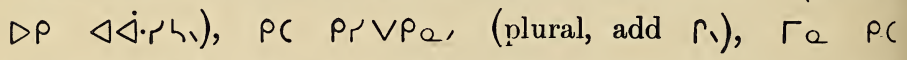

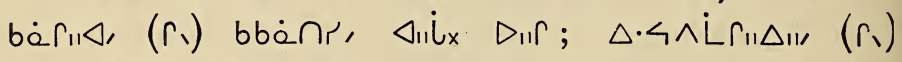

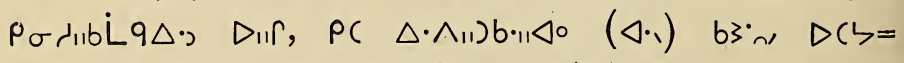

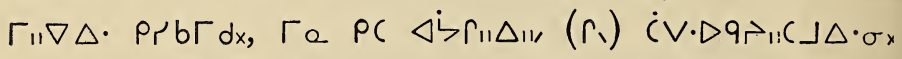

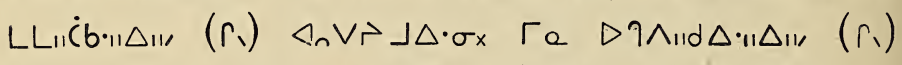

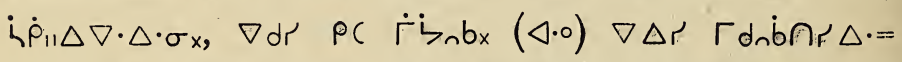

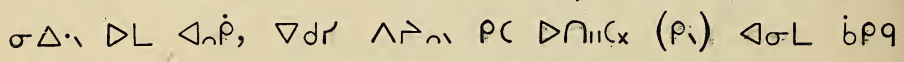
$\wedge \dot{L} \cap r \Delta \cdot \sigma \Delta \cdot \quad \Delta_{n} \dot{P}, \quad \nabla d C \quad P C \Delta \cdot \eta_{n} \quad\left(p_{1}\right), \quad \nabla \dot{b} \quad q \quad>_{\sigma}$

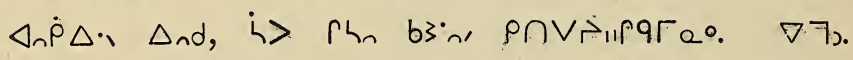

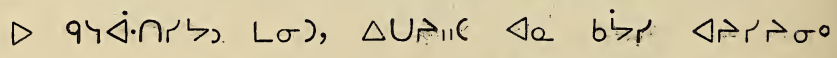

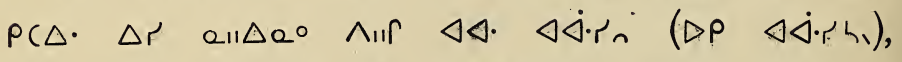

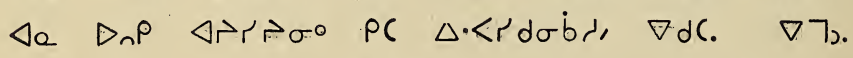

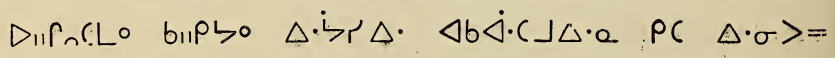

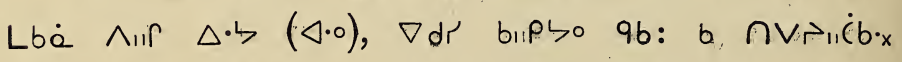

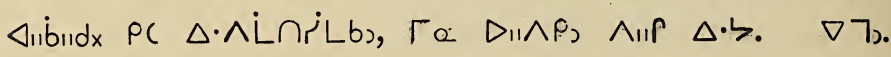


$\check{\jmath}$

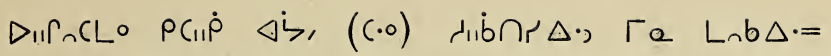

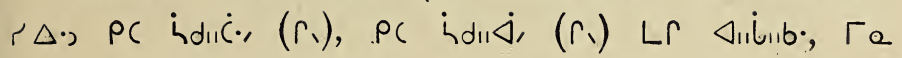
$\Delta L \quad \triangleleft_{n} \dot{\rho}$, Te $\Delta L \Delta \cdot \dot{\zeta}$. $\left.\nabla 7\right)$.

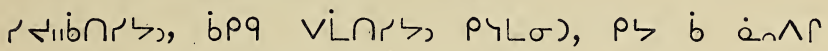

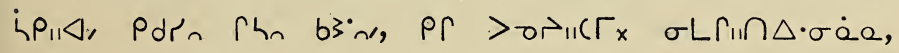

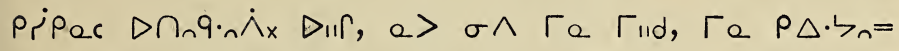

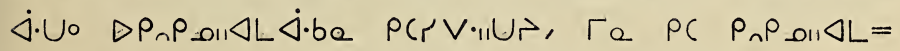

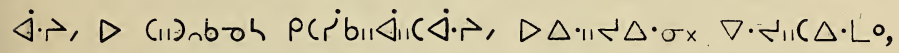

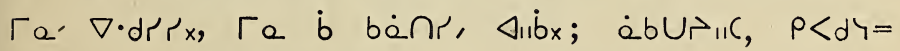

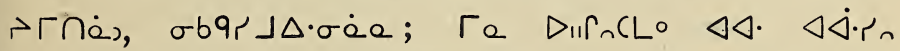

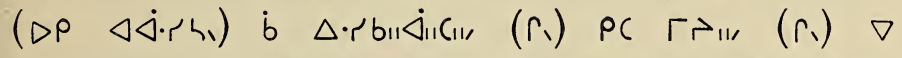

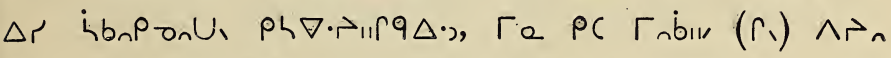
$7 \dot{b}: \nabla_{x} \triangleleft_{\sigma} \rho \quad \rho \dot{C} V \cdot \Delta \cdot \sigma \Delta \cdot, \quad a \triangleleft \cdot \lambda \dot{a} b \sigma \Gamma \quad \Delta \dot{j} \cdot r \dot{r} L_{1}, \quad \dot{h}$

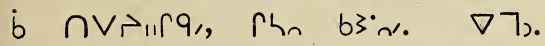

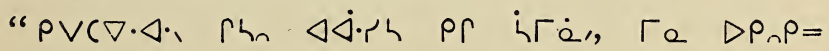

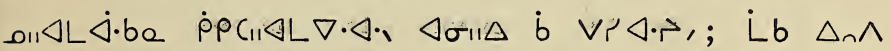

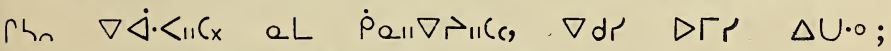

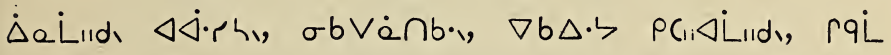

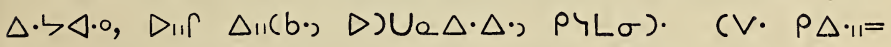
(Lne $\triangleleft \cdot 0 \wedge d \quad \Delta \Delta \cdot$ ל $\left.\nabla \dot{b} \quad 9 D \cap_{e} L q . \quad D\right) U_{e} \Delta \cdot \Delta \cdot$, $p \backslash L \sigma)\left(\Lambda_{n} d-\Delta \Lambda_{n}\left(\dot{\nabla} \cdot r_{n}\right.\right.$ eL $\left.P C_{11} \dot{\rho} \Lambda_{11}\right) q_{0} \nabla d C . "$

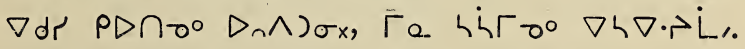

( $\nabla d C \nabla b \cdot \triangleleft ל \Gamma_{\Perp} \nabla \Delta \cdot \nabla_{\sigma}$ PC $\left.\triangle U \cdot \circ:-\right)$

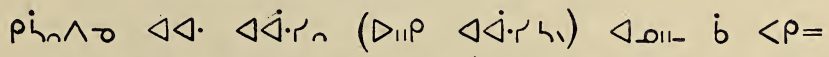

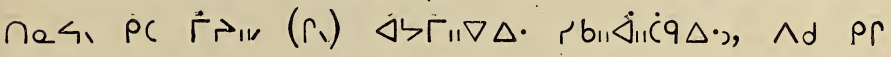

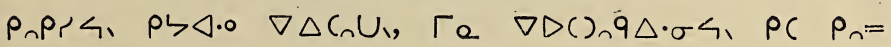


6

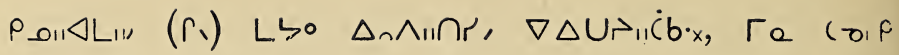

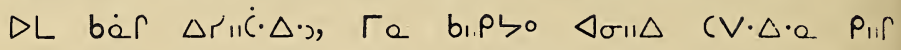
$\left.L_{r} a_{11} \Delta b \sigma x \quad d P_{n} q_{11}\left(J_{11} \Delta b \Delta \cdot b_{x} \quad p h L \sigma\right) \quad \Delta L \Gamma\right)_{0} r_{11} b_{2}$

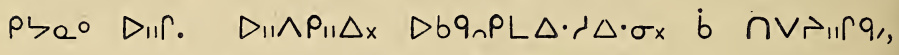

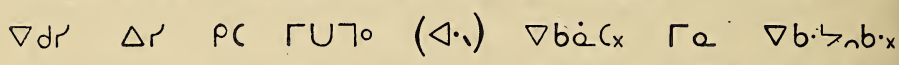

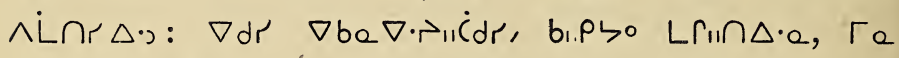

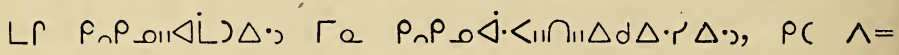

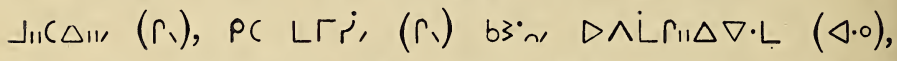

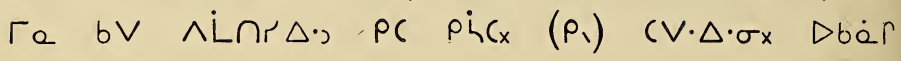
$\Delta \succ \Gamma{ }_{\|} \nabla \Delta \cdot b \Gamma \mathrm{dx}$.

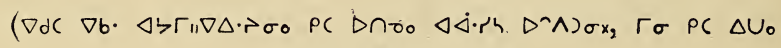

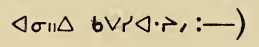

$\Delta \cdot \nabla_{x} \triangleleft \triangleleft \cdot \triangleleft \dot{j} \cdot r$

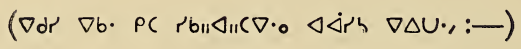

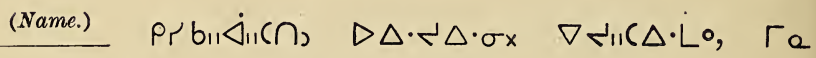

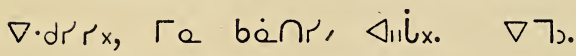

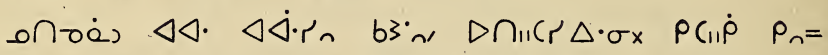

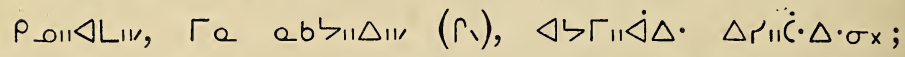

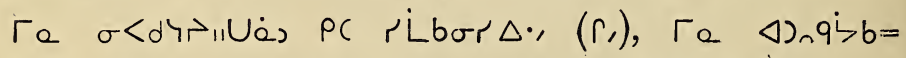

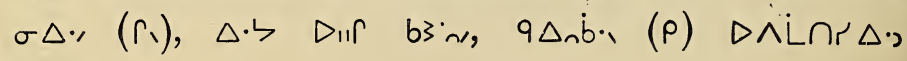
$(\sigma \triangleleft \cdot 0)$.

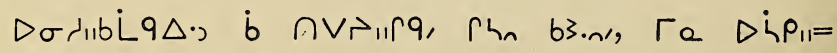

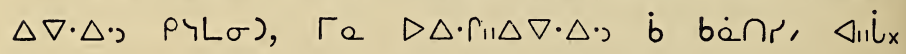
$\rho \quad b \Delta \cdot \Delta \cdot \eta \Delta \cdot d e \triangleleft \cdot 0$ bı 
7

BAPTISM OF ADULTS.

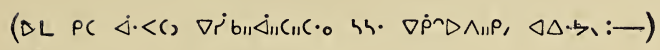

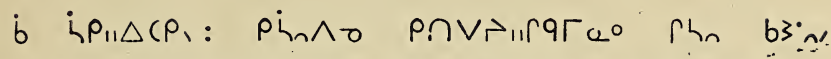

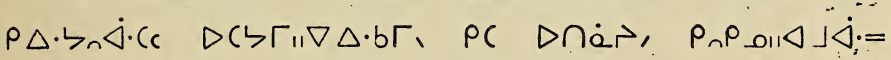

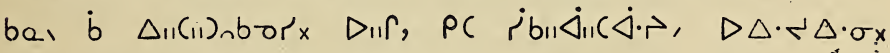

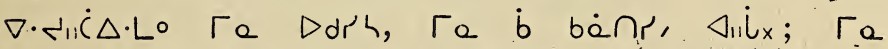

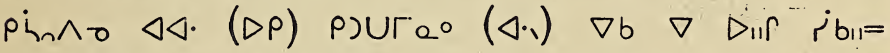

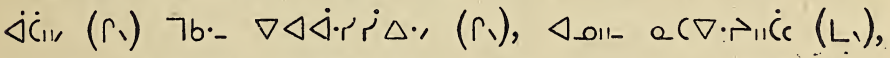

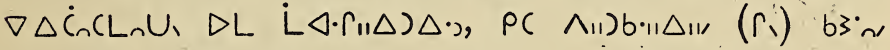

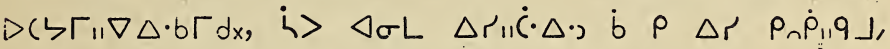

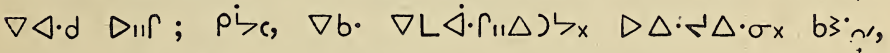

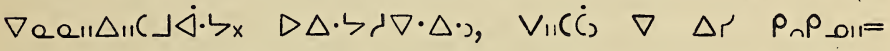

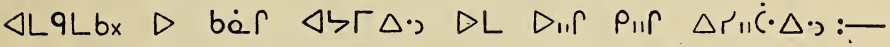
"re rho $p \vee \dot{A} p_{n} b \cdot \cup 0 \quad \nabla \Delta \dot{C}$, bu

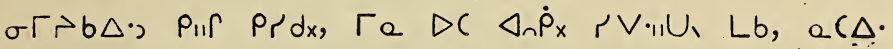

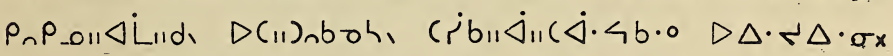

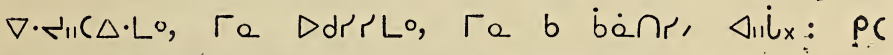

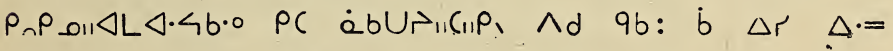

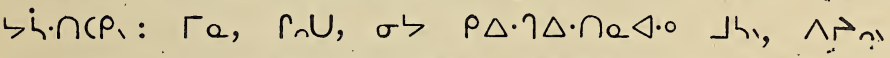
$\triangle$ nd $P C>_{\sigma} \Delta_{n} \dot{P} \Delta \cdot$. $\quad \nabla 7_{\text {o. }}$ " (Matt. xxviii. 18-20.)

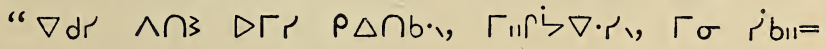

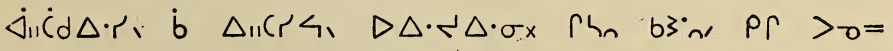

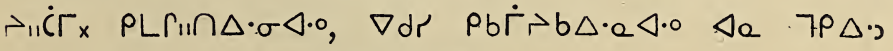
bu beans, $\triangleleft_{11} i x_{x} "$ (Acts ii. 38.) 
8

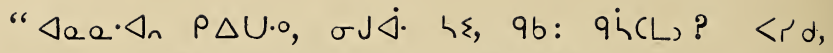

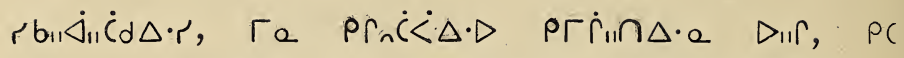
$U \dot{<} \cdot(L) \quad \nabla \Delta \cdot S \Delta \cdot)$ bi $\cap \vee r_{1}, \rho 9, "$ (Acts xxii. 12-16.)

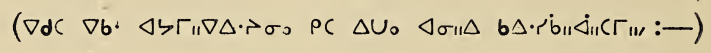

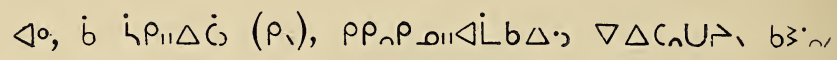

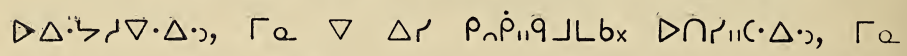

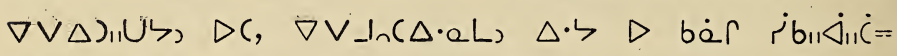
$q \Delta \cdot), \quad \nabla d C \quad \nabla \cdot \Gamma\left(\Delta \cdot \quad \rho \triangle \|_{U}, \nabla \Delta \dot{C}_{n}(L \wedge, \quad \rho Y L \sigma) \Gamma \mathrm{e}\right.$

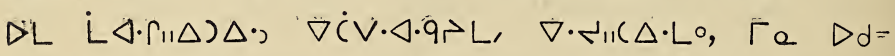

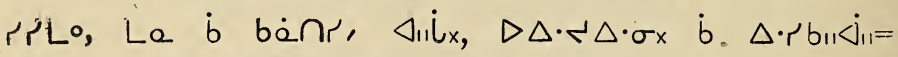

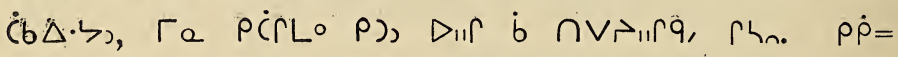

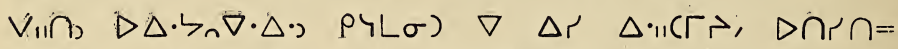

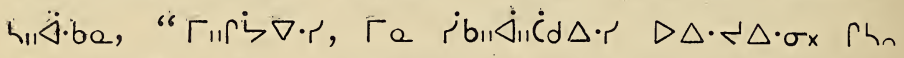

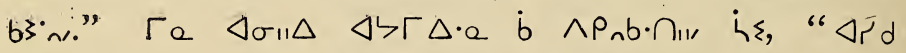

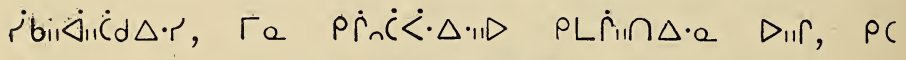

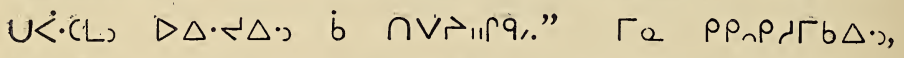

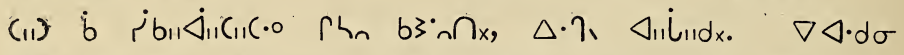

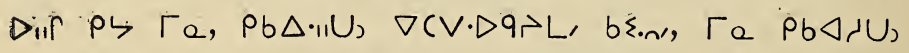

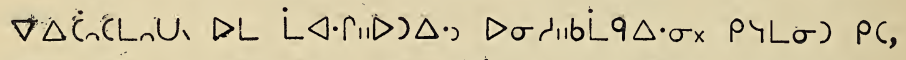

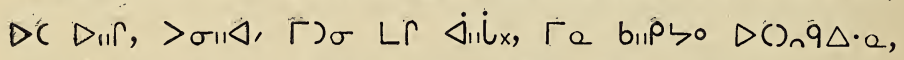

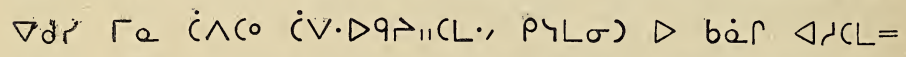

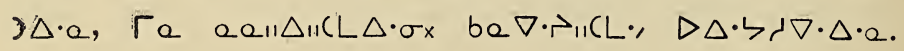

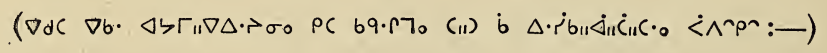

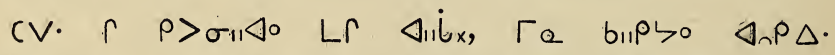

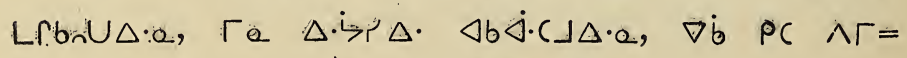

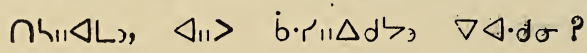


9

b॥P $>0 \quad \nabla \triangleleft \cdot \dot{d} \sigma \quad \sigma<P \cap 0)$.

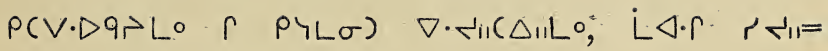

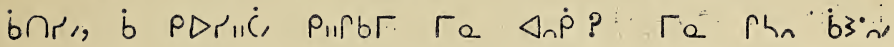

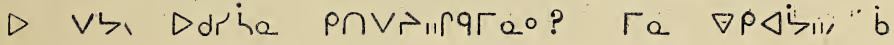

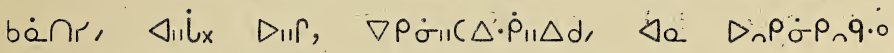

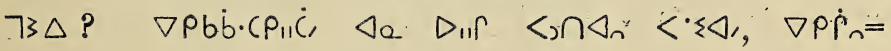

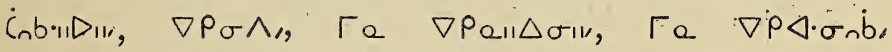

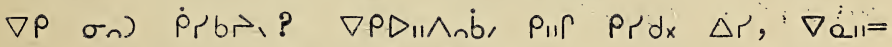

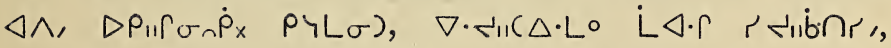
$\nabla d C$ DIIf $\dot{b} \Delta \cdot P(C d r)>_{\sigma} \Delta_{n} \dot{P} \triangle \cdot P \cdot P C \quad \triangle \cdot \zeta_{n} \dot{\nabla} \cdot \dot{C}, \dot{b}$

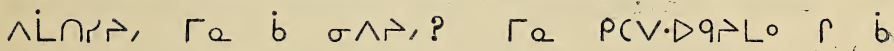

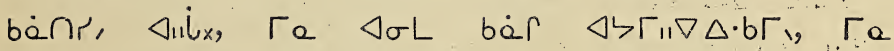
$\left.\Delta \Delta \cdot \eta \dot{\Delta} \cdot b \sigma_{11}\right) \Delta \cdot \sigma \Delta \cdot 0 \quad \Delta b \dot{a} \Gamma_{11} \Delta \triangleleft \cdot 1 ; \Gamma_{a} \quad \nabla>{ }_{0} r_{11}\left(\Gamma_{x}\right.$

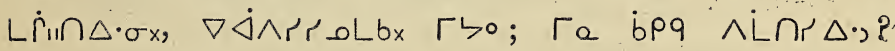
bIPᄂO $D L \quad \nabla \cdot \Gamma\left(\Delta \cdot \sigma\left(V \cdot D q \gg_{11} U\right)\right.$.

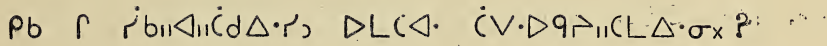
$\nabla d r^{\prime} \quad \sigma \cap r^{\prime} \Delta \dot{b} \dot{j} \cdot u_{\text {. }}$

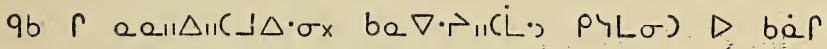
$\left.\Delta U_{\Gamma_{11}}(\lrcorner \Delta \cdot{ }_{3}, \Gamma_{e} \Delta \cdot \zeta_{n} \nabla \cdot \Delta \cdot a, \Gamma_{\sigma} \wedge J_{11} \dot{C}\right), \nabla \Delta \cdot d \sigma$

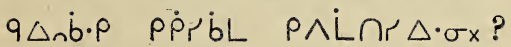

$$
\sigma b b q \cdot) U, \quad P Y L \sigma) \sigma(\| b\lrcorner \Delta \cdot \Gamma \text {. }
$$

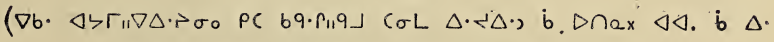
Íb: $\Delta C_{\text {HII }}, \nabla d r \quad \nabla \triangleleft \cdot d$ PC $\triangleleft<r_{11} C_{0}$ PC $\left.\Delta U \cdot,:-\right)$

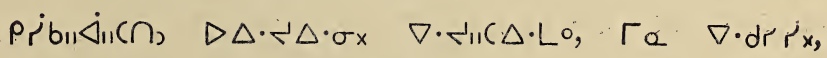

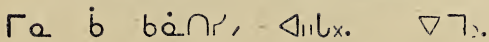


10

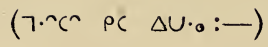

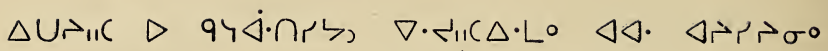
$\left(\Delta p \quad \Delta r_{r} \nabla_{\sigma} \triangleleft \cdot 1\right) \quad \triangle$ ـ

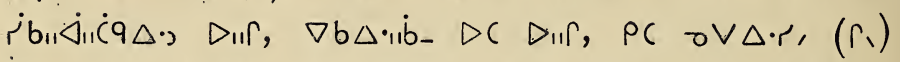

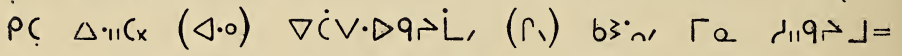

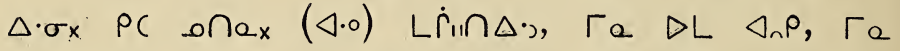

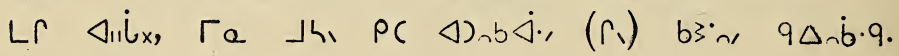
$\Delta \wedge$ LnR $\triangle \cdot, \quad(\sigma \triangleleft \cdot 0) . \quad \nabla 7)$.

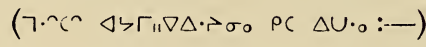

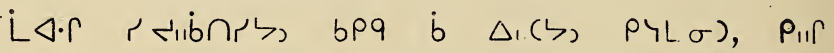

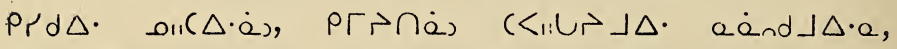

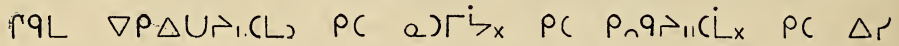

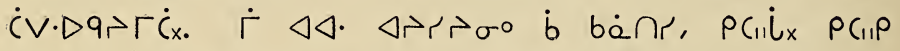

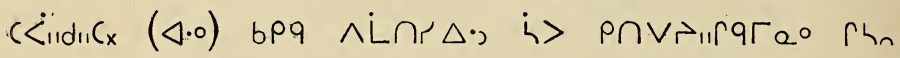

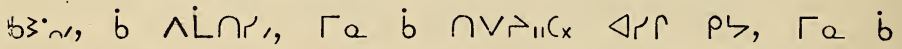

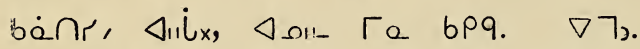

$\left.D_{11} \dot{\Gamma}_{n} C L \circ b_{11} \rho L_{0} \Delta \cdot \dot{L}_{r} \Delta \cdot \quad \Delta b \dot{j} \cdot \cap\right\lrcorner \Delta \cdot a, \quad \rho C \quad \sigma>=$

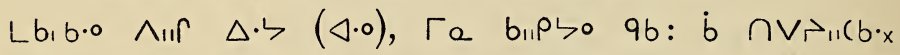

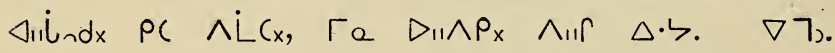

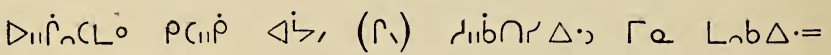

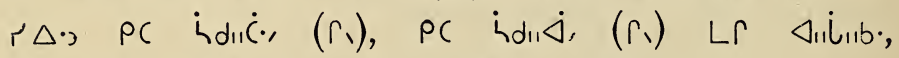

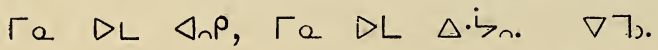

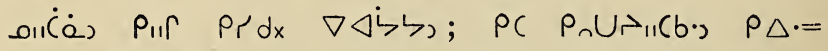

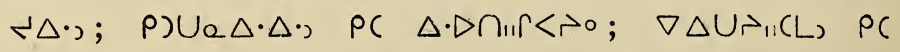

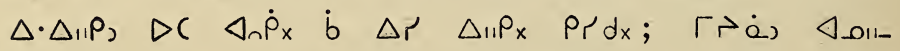

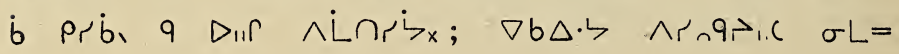


11

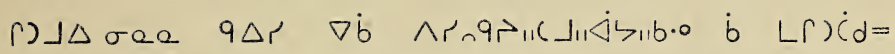

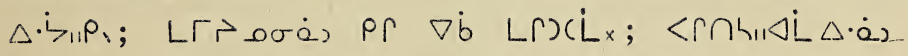

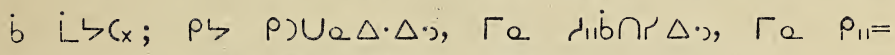
ir $\rightarrow \Delta \cdot \Delta \cdot$, bpq, re bpq. $\nabla 7$.

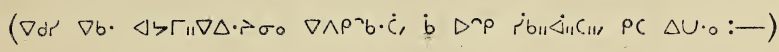

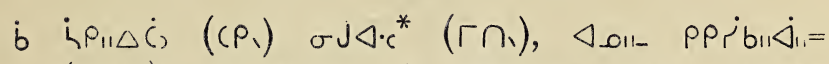

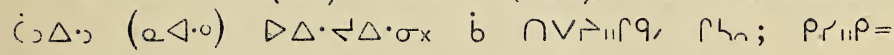

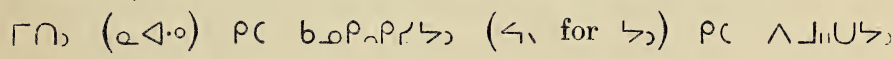
(4, for ל) $\nabla \Delta \dot{C}_{n}\left(L \dot{<} U_{1} \dot{b} \Delta \dot{<}_{1}, \Gamma a\right.$ b $b \dot{a} C_{x}$

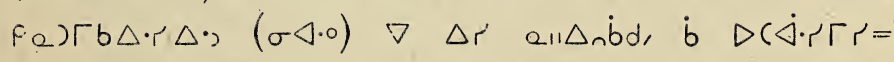

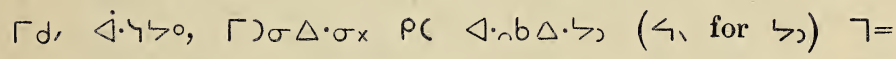

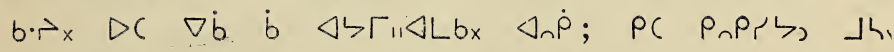

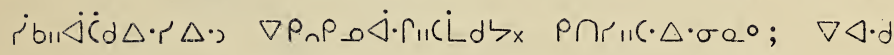

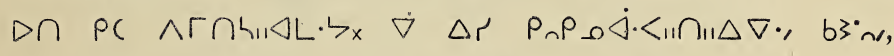

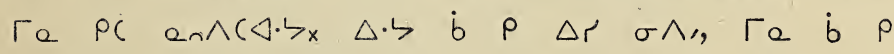

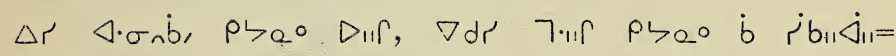

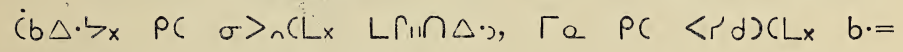
לnpini $\triangle \cdot$ ); bPq $p C$ bq. $\sigma<_{11} b_{x} p$ Lr $\left.\Delta b \dot{j} \cdot C\right\lrcorner \Delta \cdot=$

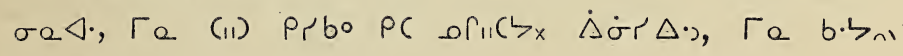
$\wedge \mathrm{L} \cap \mathrm{A} \Delta \cdot$

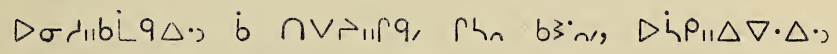

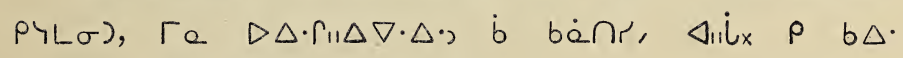

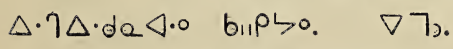

* The feminine word is $\sigma \odot \nabla \cdot L^{\circ}(L \cap)$. 
12

II.

RECEPTION OF MEMBERS.

$\left.\nabla \wedge_{11}\right) b \cdot \| \Delta_{\|}\left(\cdot 0 \quad D_{n} p \quad \Delta\left(\zeta \Gamma_{11} \dot{\Delta} \triangleleft \cdot\right.\right.$.

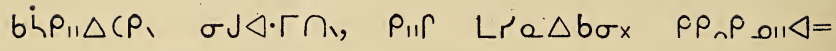

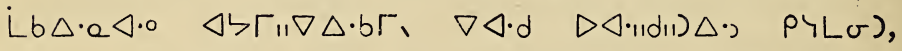

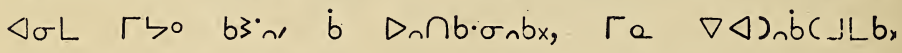

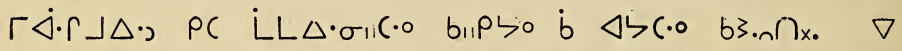

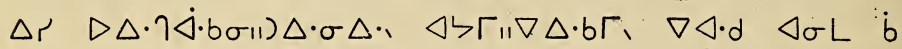

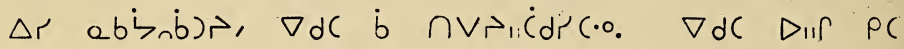

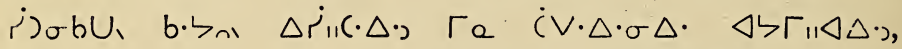

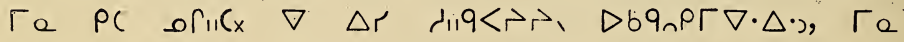

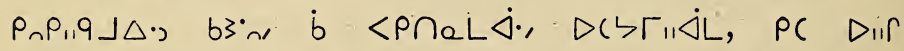
$D_{11} \wedge p_{x}$ be ami $\triangle \cdot$.

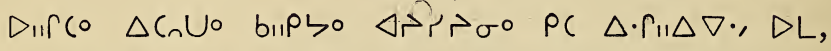

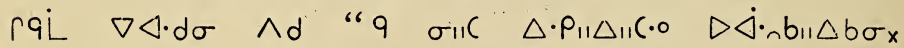

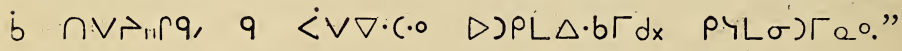

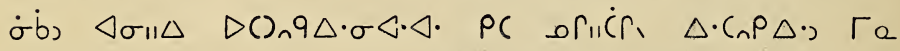

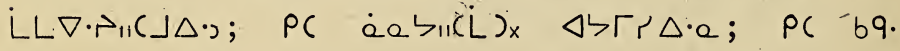

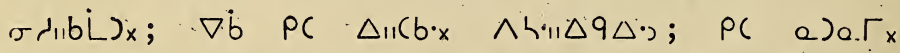

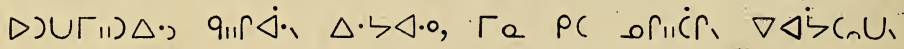

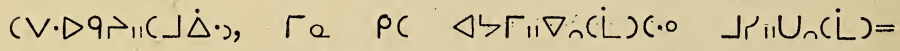

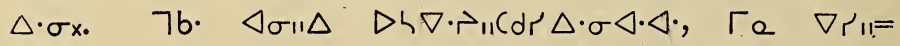

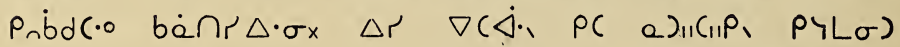


13

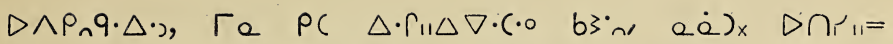

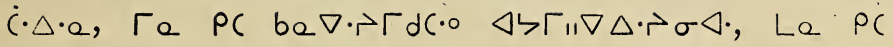

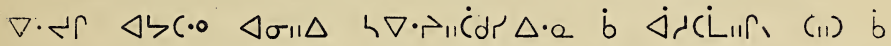

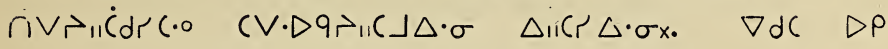
$P \cap \dot{C}_{n}\left(L \wedge \Delta \cdot \sigma \dot{\triangleleft} \cdot x \quad \vee \perp_{n}(\Delta \cdot 0 L \cdot\right.$. $P C \wedge) 9(\cdot 0$.

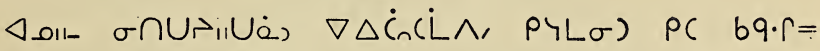

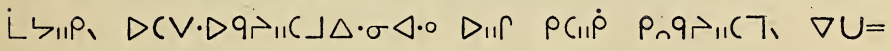

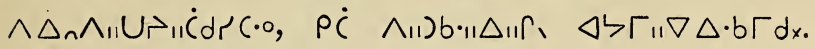

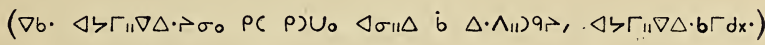

b $\quad$ i $p_{11} \Delta(p, p \vee \triangle)_{11}(Q \triangleleft \cdot 0 \quad D C \quad \nabla \triangleleft b \triangleleft \cdot C 7 、$ PC $\Delta \cdot=$

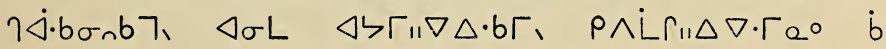

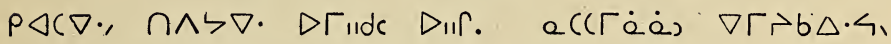

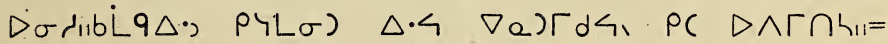

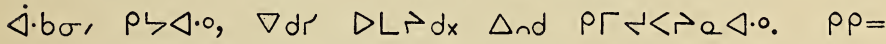

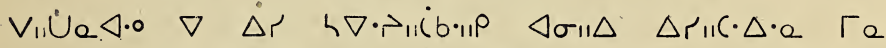

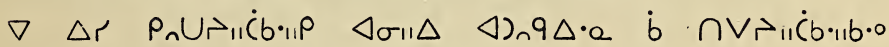

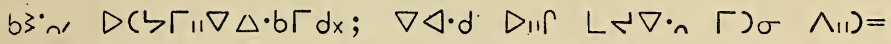

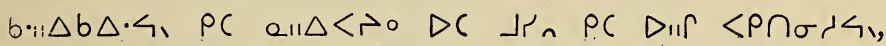

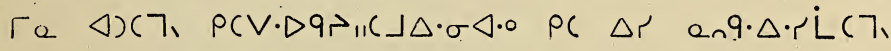
বـoll 9 b9.r厂b $\Delta \cdot 4:-$

Pb $\Gamma \Delta C \nabla \Delta \dot{C}_{n}(\Gamma \wedge, \quad \rho Y L \sigma)$, Ta $D L L \dot{j} \cdot \Gamma_{11}=$

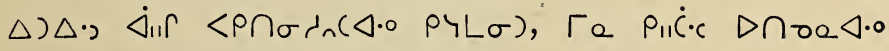

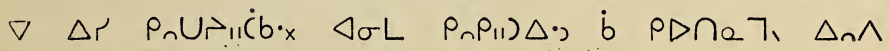

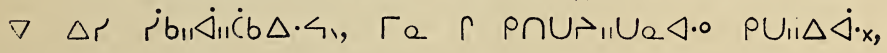
$p \wedge i \cap \Delta \cdot \sigma \dot{\psi} \cdot x, \quad \sigma>\Delta \cdot \sigma x, \quad \Gamma_{e}$ bpq $P C, \quad\left(V \cdot \Delta \cdot \sigma_{x}\right.$, $\triangleleft), b \triangleleft 4, \quad p(L) \sigma)$ ? 
14

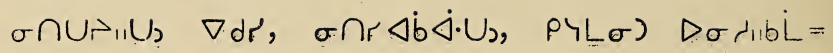
$9 \triangle \cdot \sigma \times \quad D_{11}$.

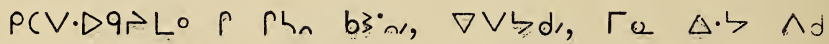

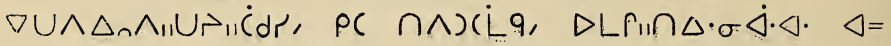

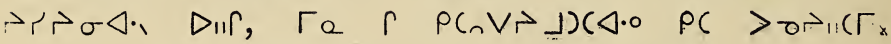

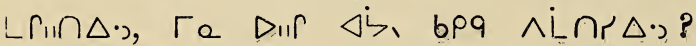

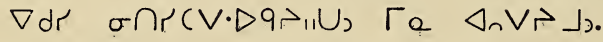

$p\left(V \cdot D q P_{11}\right) \Gamma$ PYLo) $\nabla r \| P \cap b x$ ro $\Delta \cdot ל \quad D=$

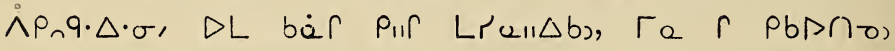

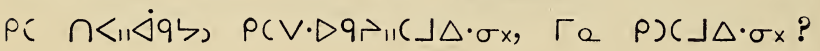

$\nabla ॥ \nabla, \quad \nabla d r \quad \sigma) U$.

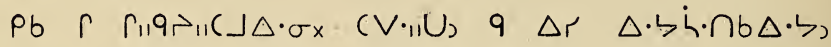

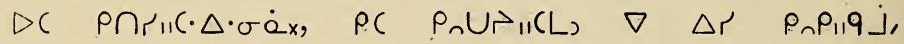

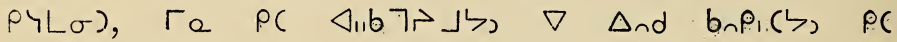

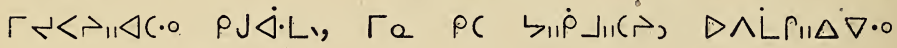
$\left.\sim \cup_{a} \triangle \cdot \Delta \cdot\right)$ ?

$\sigma b) U$.

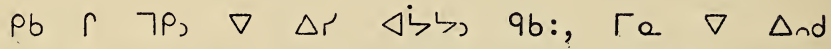

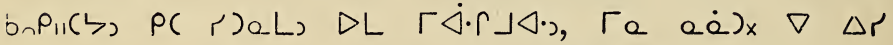

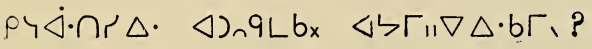

$(\sigma-b) U$.

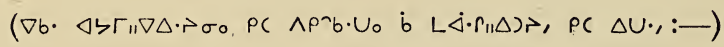

б)U

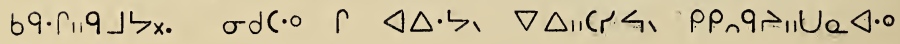


15

96: $\left.\nabla \dot{b} \quad 9 \dot{\rho} \quad D_{11} \Gamma \quad \Lambda_{11}\right) b \cdot 1 \Delta_{11}\left(\cdot 0 \quad \Delta ל \Gamma_{11} \nabla \Delta \cdot b \Gamma d x \quad P C\right.$ Г) $\sigma \Delta \cdot \Gamma_{11} \Delta \nabla \cdot(\cdot \circ$ ?

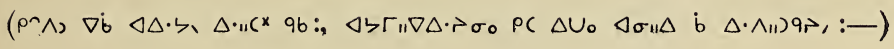

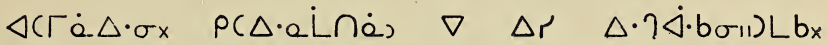

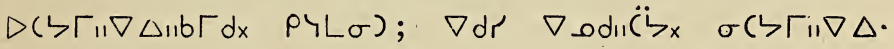

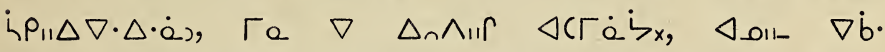

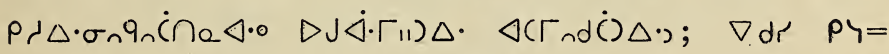

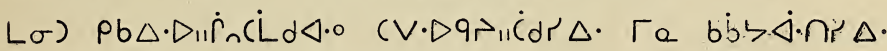

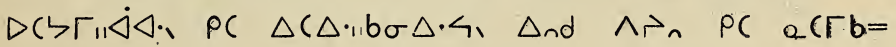

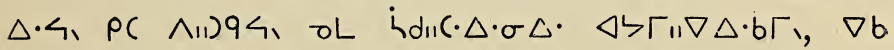

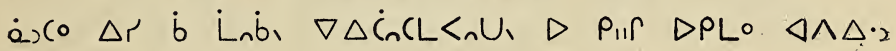
c) $(\sigma)$ ). 
16

III.

THE LORD'S SUPPER.

b nVruíq, Dbár pir $\Delta r \| \cdot \Delta \cdot \%$.

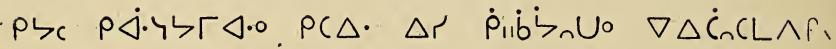

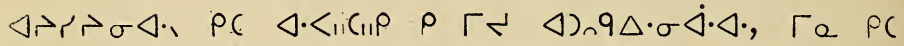

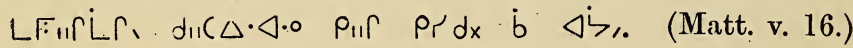

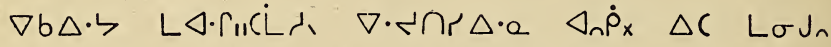

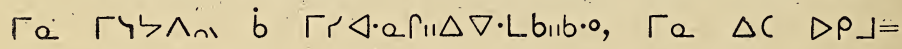

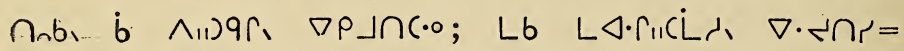

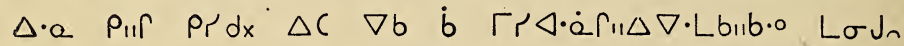

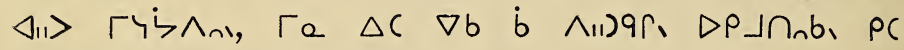
p.Jnc.o. (Matt. vi. 19, 20.)

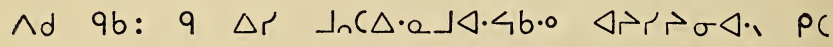

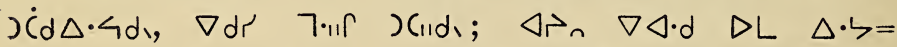
i $\nabla \cdot \Delta \cdot$, , Г० $\Delta \cdot \dot{\Delta}_{11} \Gamma 9 \Delta \cdot$, (Matt. vii. 12.)

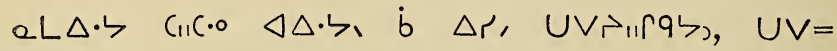

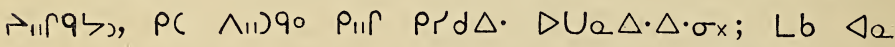
b) $x_{x} \Delta \cap \cup r_{11}$ b)

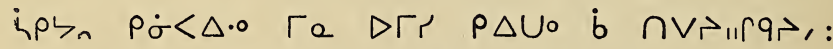

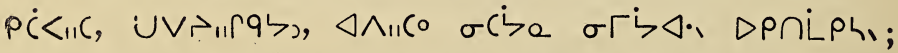

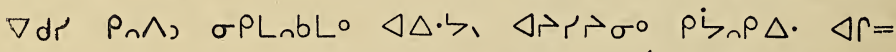
\lrcorner$\Delta \cdot$, DII $b \Delta \cdot \sigma<P \cap a\lrcorner \triangleleft \cdot \circ \quad \diamond \triangleleft \cdot \circ \Delta r_{d x}$. (Luke xix. 8.) 
17

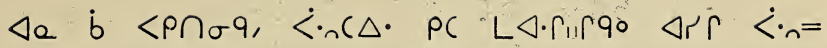

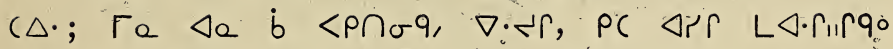

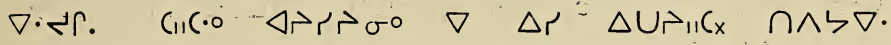

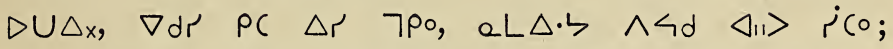

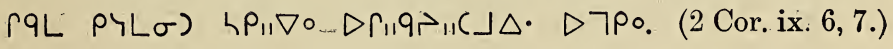

$\nabla \triangleleft \cdot d$ DiIf $\nabla \Delta$ nd $\left(\Delta \cdot a \dot{L} b \Delta \cdot b_{x}\right.$, pise $\left.\left.\Gamma<\right) \dot{x} \triangleleft \cdot \dot{C}\right)$

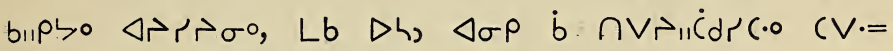
$\triangle Q_{r_{11}} \dot{C} d r \Delta \cdot \Delta \cdot \cap_{11} C_{r} \Delta \cdot \sigma x_{.} \quad$ (Gal. vi. 10.)

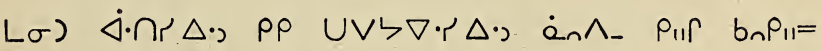

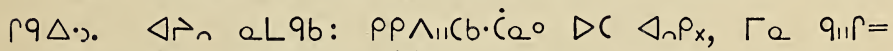

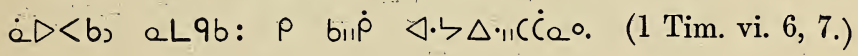

Pnplind $\Delta \sigma \rho$ bे $\nabla \cdot \Delta \cap r C \cdot 0$ DC $\triangle_{n} \dot{\rho} \quad \nabla b \quad \rho C$

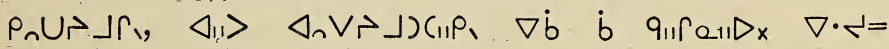

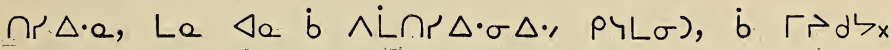
$\nabla \cdot \lambda r$ bा1

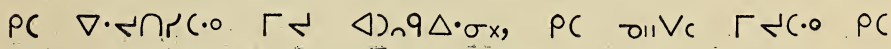

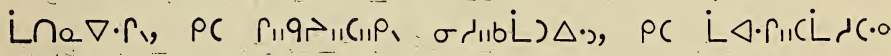

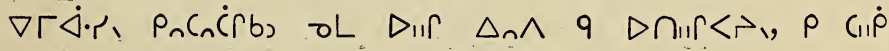
birfneıp, bpq $\wedge$ Lח $\Delta \cdot{ }^{\prime}$. (1 Tim. vi. 17-19.)

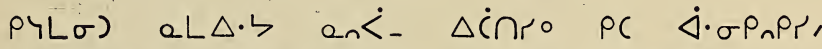

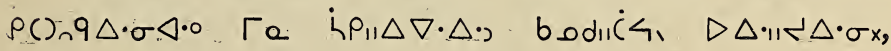

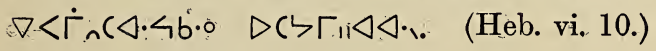

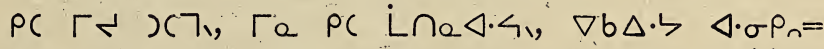

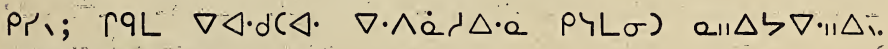
(Heb. xiii. 16.) 
18

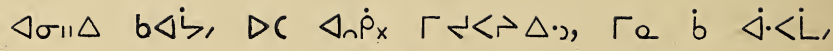

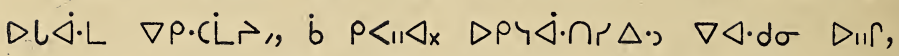

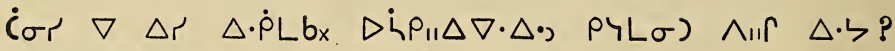
(1 John iii. 17.)

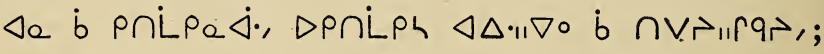
$\Gamma_{a} \triangleleft \sigma L$ b $7 P, b \triangle . P C \cap<11 L_{1} . \quad$ (Prov. xix. 17.)

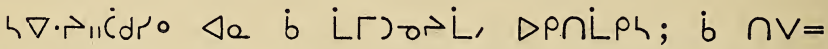

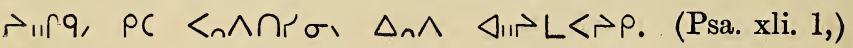

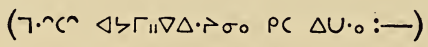

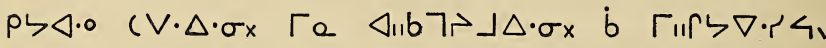

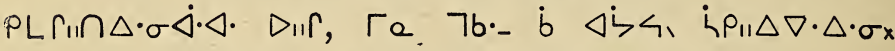

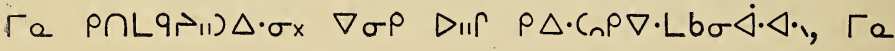

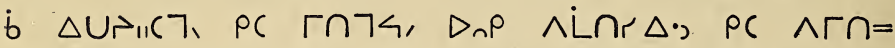

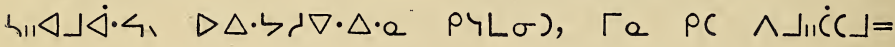

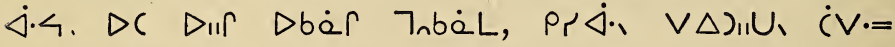

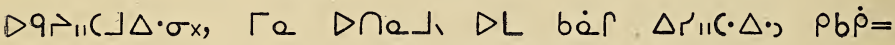

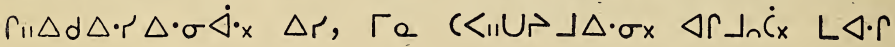
«

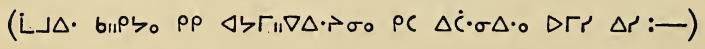

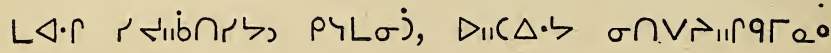

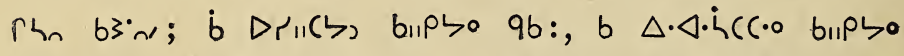

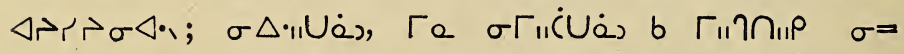

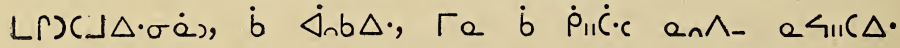
$\left.\left.x i_{x}, \quad L_{\Gamma}\right)_{0} r_{11} b_{\sigma x}, \wedge p_{n} q \cdot \Delta \cdot \sigma x, x\right\lrcorner \Delta \cdot \sigma x \nabla \Delta \dot{C}_{n} C_{n} U_{1}$ 
19

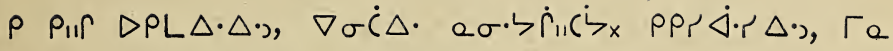

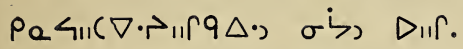

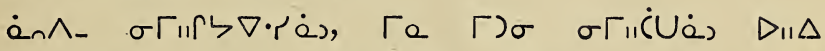

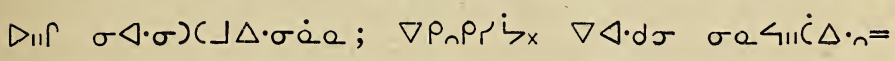

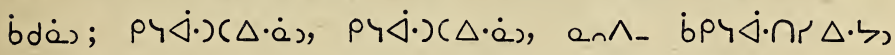

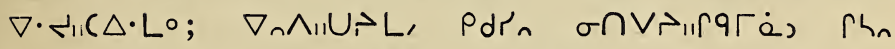

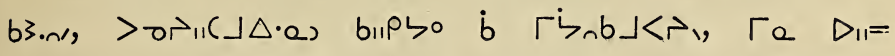

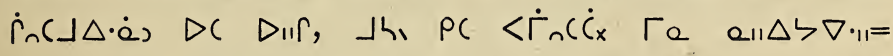

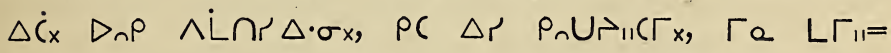

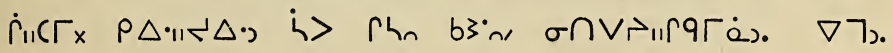

$\left(\nabla d C \nabla b \cdot p C \Delta U \cdot 0 \Delta b \Gamma \nabla \nabla \Delta \cdot \nabla_{0} \sigma_{0}:-\right)$

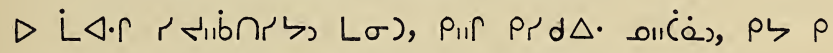

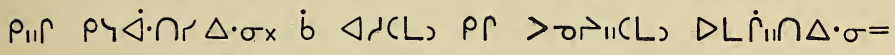

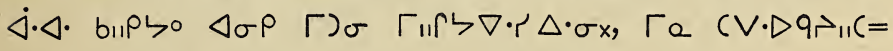

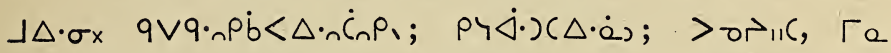
< $\wedge \cap$ r $\sigma \dot{\alpha}$ ) bו1

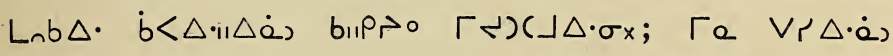

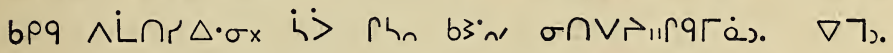

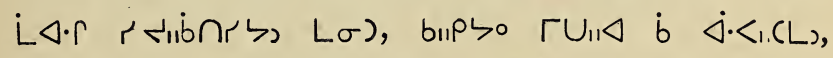
bו

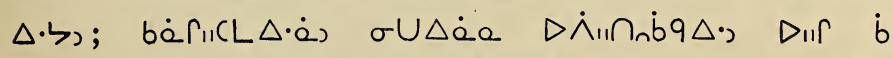

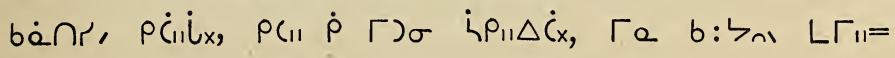

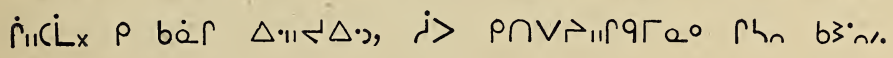

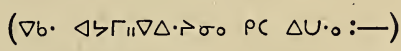

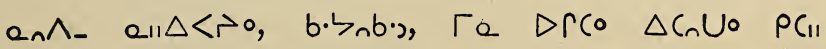

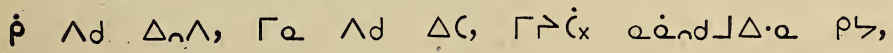


20

D UVP bpq $\left.b \quad \Delta_{11}(\zeta) \quad L \sigma\right)$;

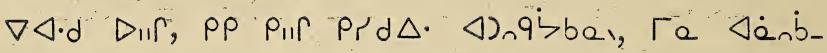

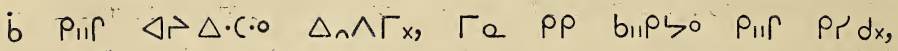

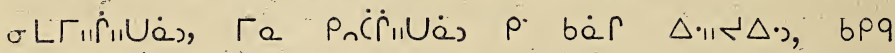
$\nabla L \Gamma$ IIR

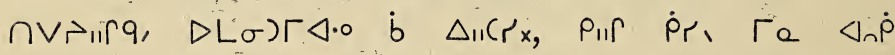

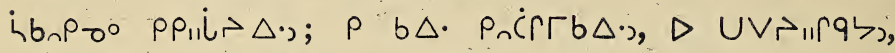

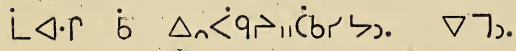

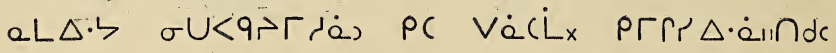

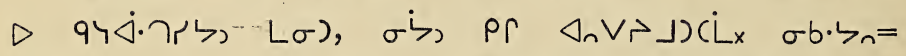

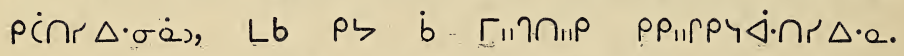

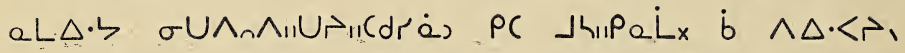

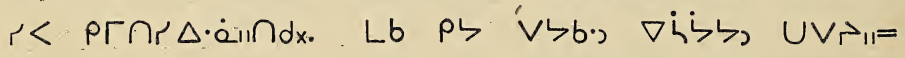

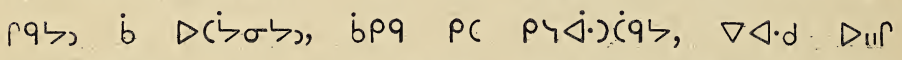

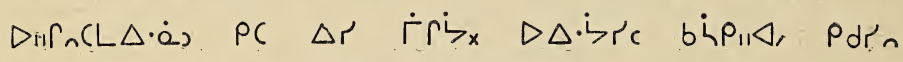

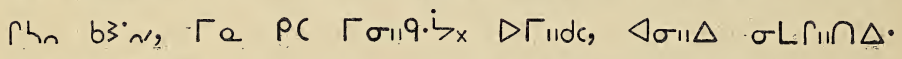

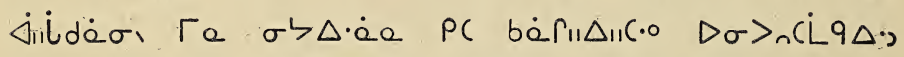
Dil, $\Gamma_{a} p C$ prVPa $\Gamma_{x}$ Dbér $\Gamma_{11 d x,} p C \triangle \cdot P L L_{x}$ DC DII bPq, Га $\triangle \cdot P \Gamma d \zeta_{x} . \quad \nabla 7$ ).

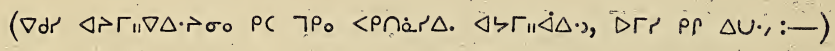

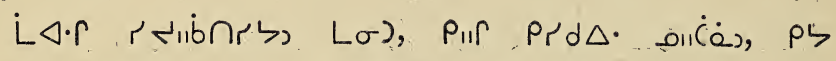

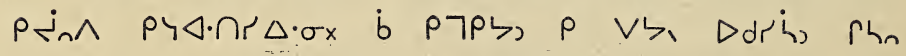

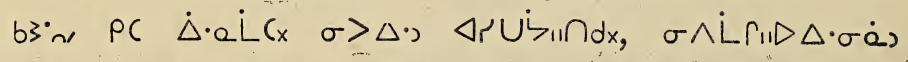

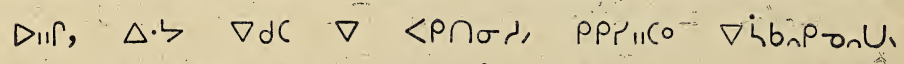

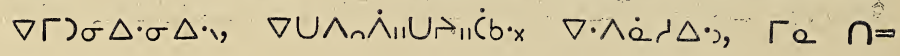


21

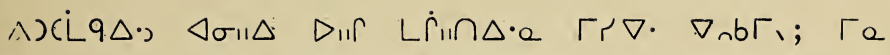

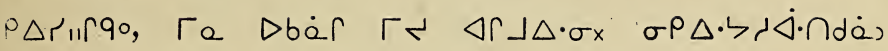

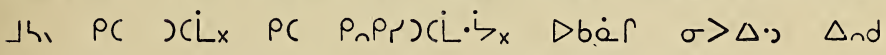

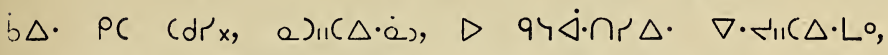

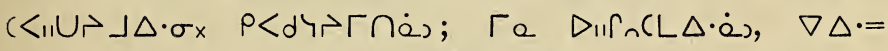

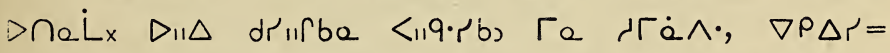

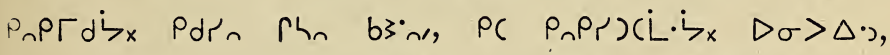

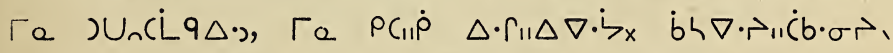
$D \Delta$.

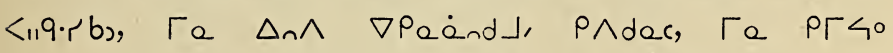

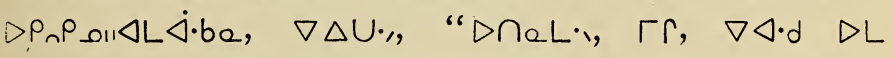

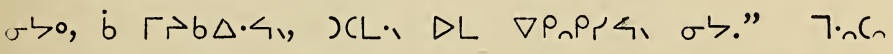

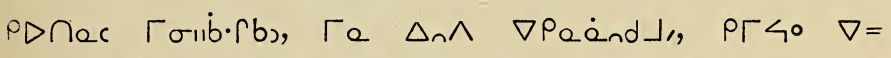

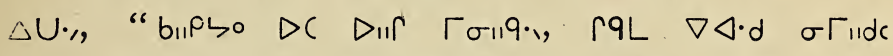

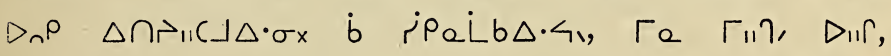
$P\left(>_{0} \rightarrow_{11}\left(\Gamma \times \Gamma_{11} \Delta \cdot 0, \Delta \Delta \cdot d\right)\left(L \cdot{ }^{\prime}, \Gamma_{0} C_{11} C \cdot 0 \quad \nabla \Gamma=\right.\right.$

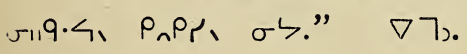

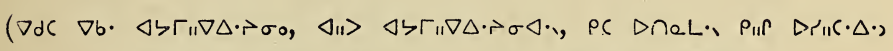

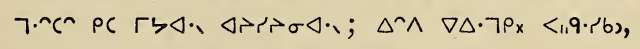
$\Delta \Gamma r$ pC $\Delta \dot{C} \cdot \sigma \Delta \cdot 0:-)$

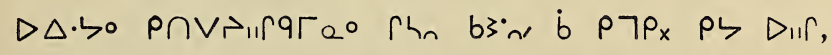

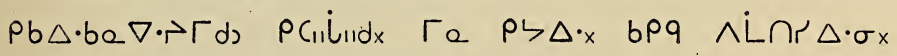

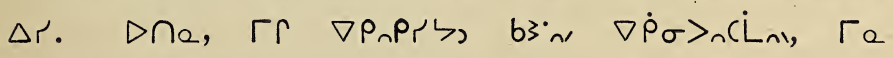

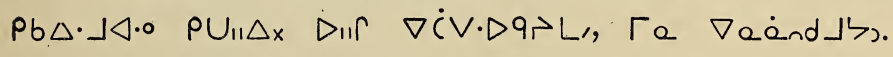

(

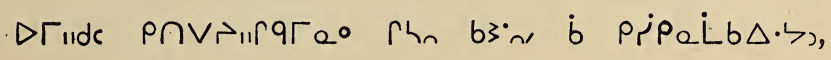


22

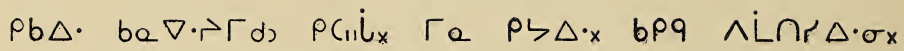

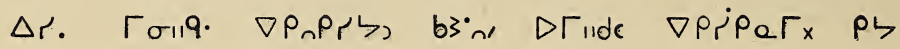

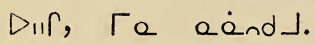

( $\left.\nabla d r P C_{11} \dot{P} \Delta \dot{C} \cdot \sigma \Delta \cdot 0:-\right)$

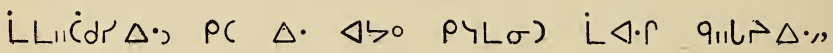

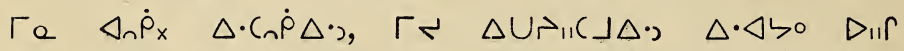

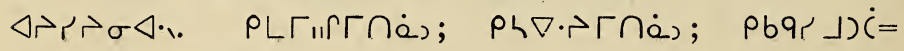

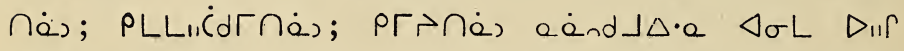

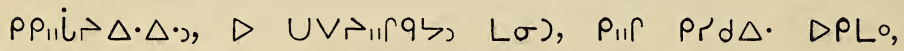

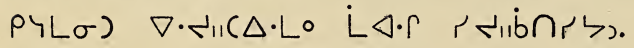

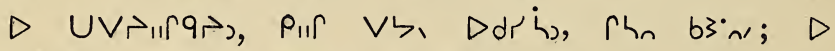

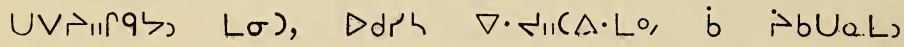

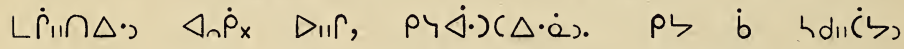

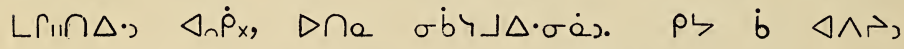

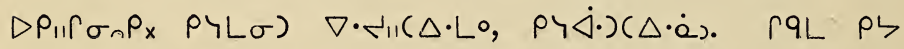

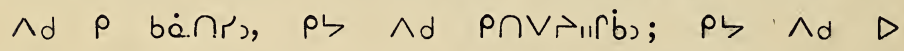

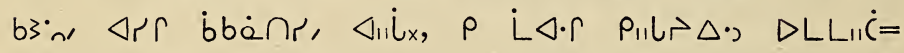

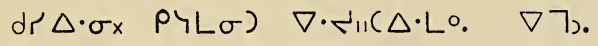

$(4 \nabla \cdot \vec{r} u \Omega \Delta \cdot \dot{\rho} \varphi \cdot \cap) \Delta \cdot))$

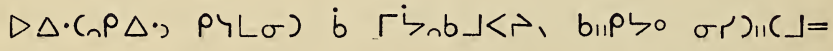

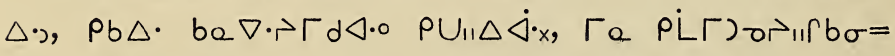

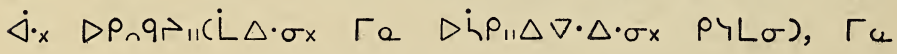

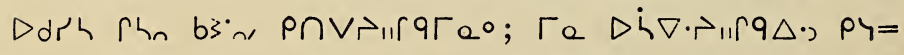

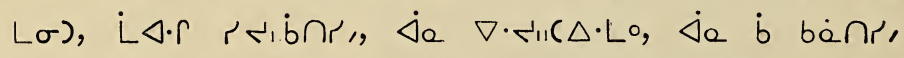
$\triangleleft_{11} i_{x}$ pb $\Delta \cdot \Delta \cdot 7 \Delta \cdot d a \triangleleft \cdot 0$ Га pinda $\triangleleft \cdot 0$ bpq. $\nabla 7$. 
23

IV.

MARRIAGE SERVICE.

$\left.p_{11 \Gamma} \quad \Delta_{11} p_{11}\right) \Delta \cdot{ }^{\prime}$

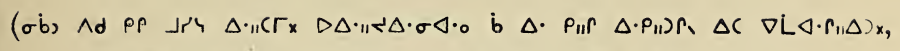

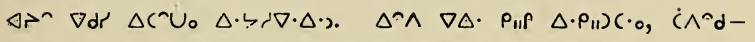

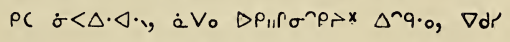
$\Delta b \Gamma_{\| \prime} \nabla \Delta \cdot r^{\circ} \sigma_{0} b\left(\Delta U_{0}:-\right)$

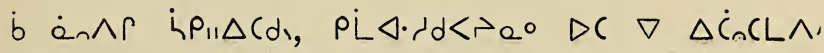

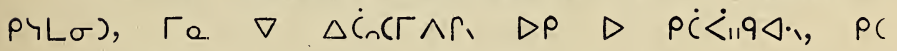

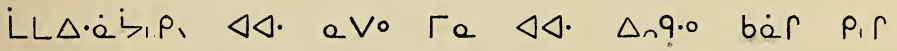

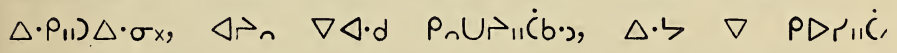

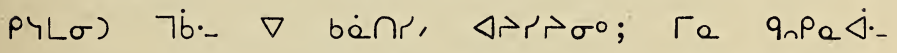

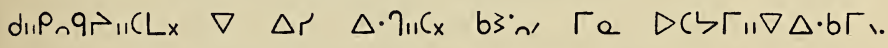

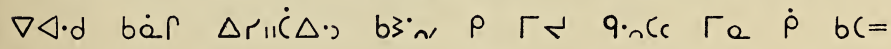

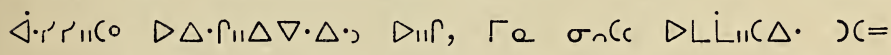

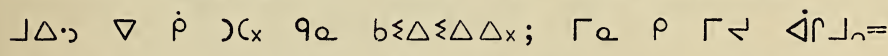

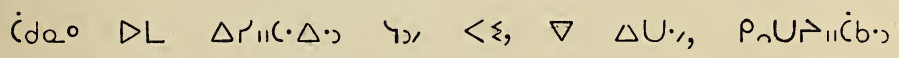

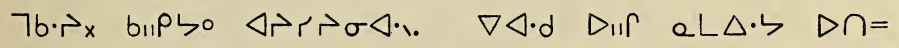

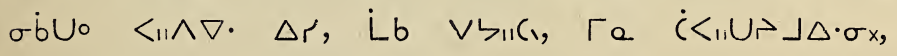

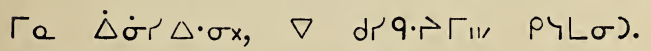

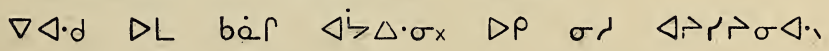

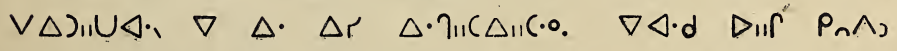

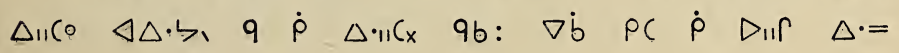


$2 t$

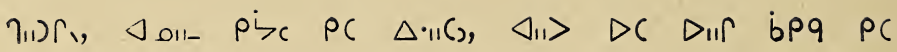
pisc $\triangleleft_{n}(0 \quad \nabla \wedge P \cap q \cdot \Delta \cdot)$.

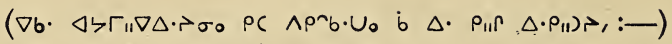

pec

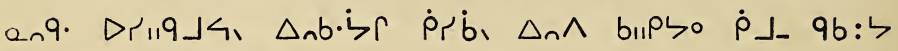

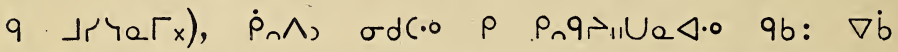

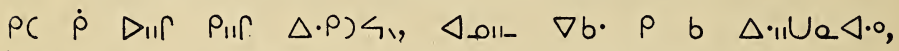

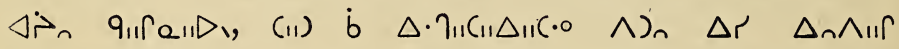

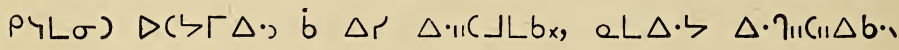

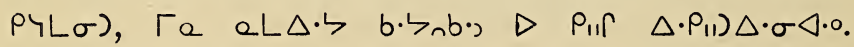

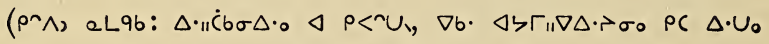

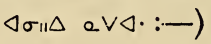

(Name.) Pb $\dot{\Gamma} \triangleleft ৬ \triangleleft .0 \triangleleft \triangleleft . \quad \Delta, 9.0$ PC PIII $\triangle . P L \prime$,

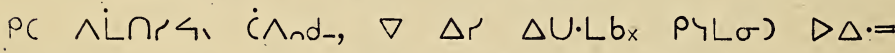

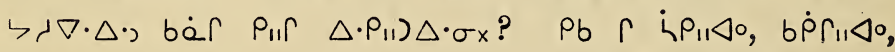

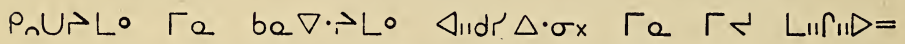

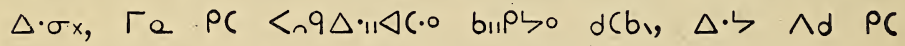

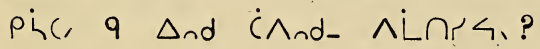

$(\nabla d r$ eVo oC $\Delta U \cdot 0:-)$

ob)U.

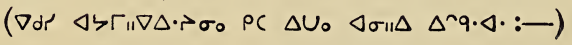

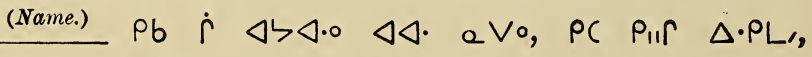

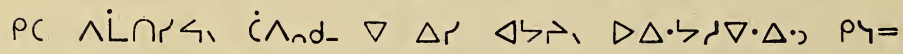

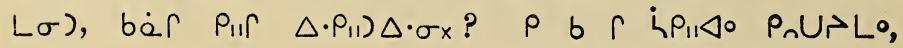


25

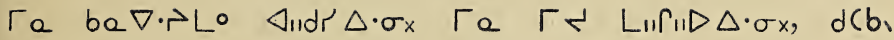

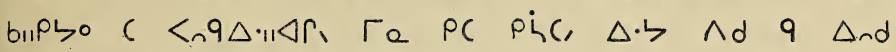
$\wedge$ Lnori ind_?

$\nabla d r \Delta\urcorner \cdot \circ P(\Delta U \cdot 0:-)$

$\sigma b) U_{2}$

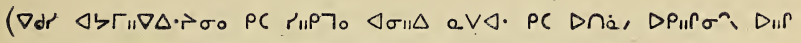

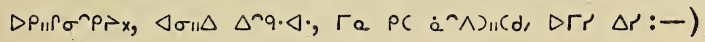

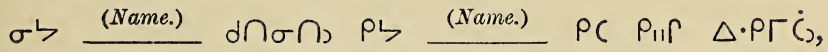

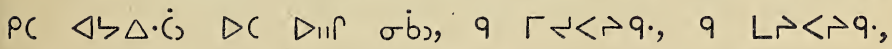

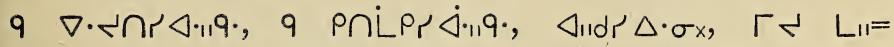

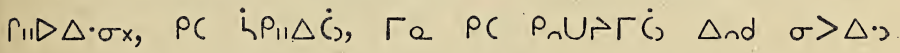

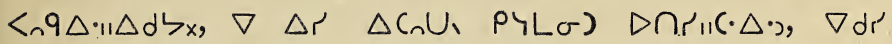

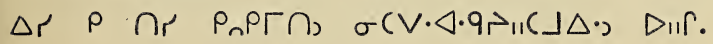

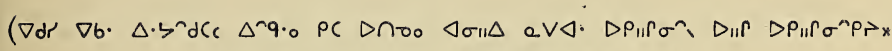

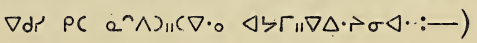

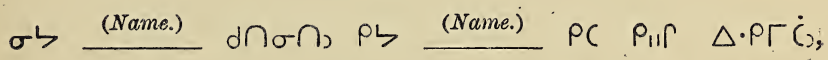

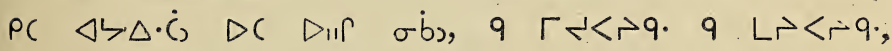

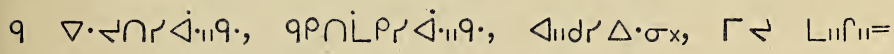
$\Delta \Delta \cdot \sigma x, \quad P\left(\quad p_{11} \Delta \dot{C}\right)$, Ta $P\left(P_{n} \cup r \Gamma \dot{C}\right) \quad \Delta n d \quad \sigma>\Delta \cdot$.

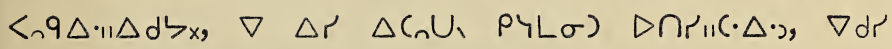

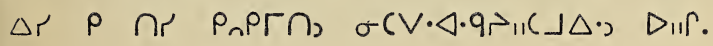

$\triangleleft ৬ \Gamma_{\|} \dot{\jmath} \dot{c}$

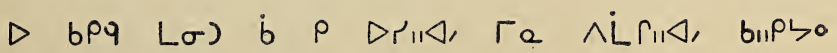

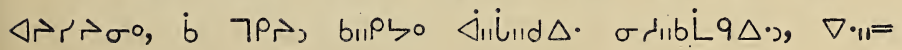


26

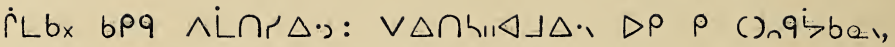

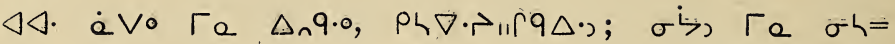

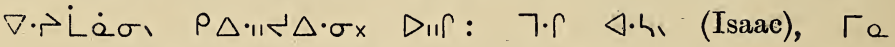
s $\Delta \vee b \quad$ (Rebecca), ib $\left.\dot{\rho} \quad \Delta r \quad \dot{C} V \cdot \Delta \cdot \sigma x \quad \Delta \cdot \eta_{11}\right) r, \quad \nabla d r$ $\triangle P \Delta r_{r} \nabla_{\sigma} \Delta \cdot, P C \Delta \cdot \Delta r$ be $\nabla \cdot \nabla_{11} C L \cdot \Delta \sigma L P_{n} P_{11}=$

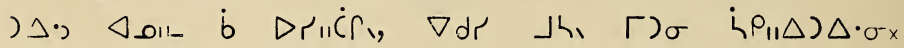

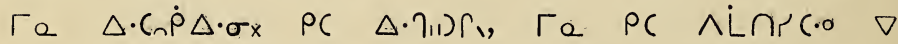

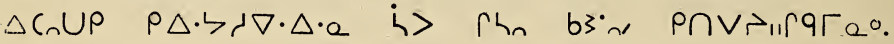
$\nabla 7$.

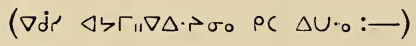

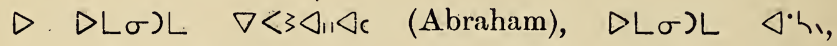

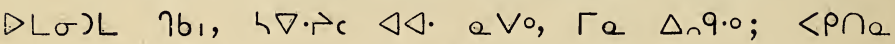

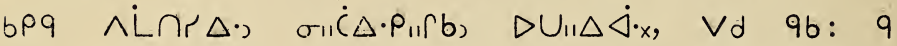

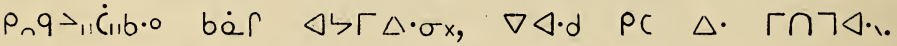

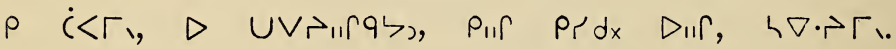

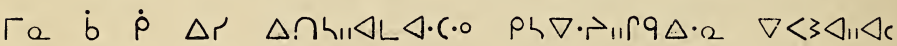
Ta $4 \Delta \Delta \Delta \dot{b} \dot{P}_{11} \Delta d \Delta \cdot r \Delta \cdot \sigma \dot{j} \cdot x \quad \Delta r, \quad \nabla d r \quad \Delta r \quad \Delta h_{11}=$

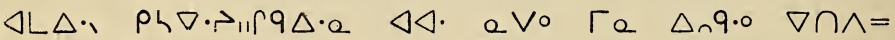

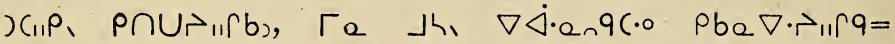

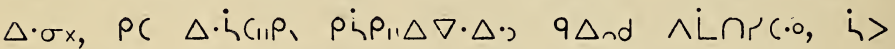

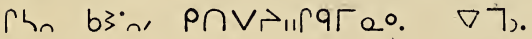

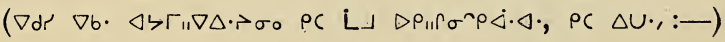

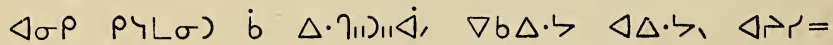
$r_{\sigma^{\circ}} P C$ QQ் $\sigma \cap \cap \sigma^{\circ}$.

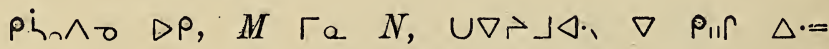
$\left.\rho_{11}\right)\left(\cdot 0, \Gamma a\right.$ odil $\dot{C} \cdot 1, \nabla \triangleleft \cdot d \nabla \Delta \dot{C}_{n}\left(L \wedge \curvearrowright, \quad \rho L^{\prime} \sigma\right), \Gamma a$ 
27

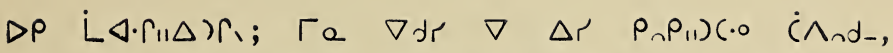

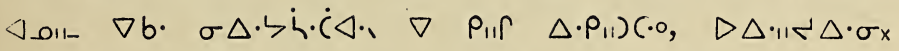

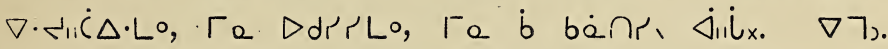

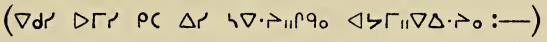

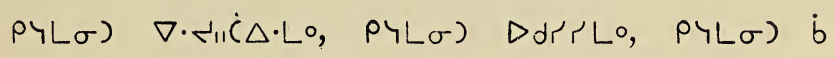

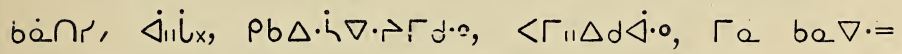

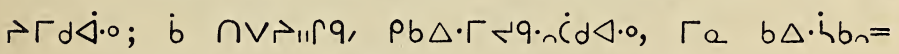

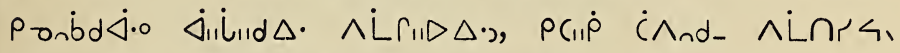

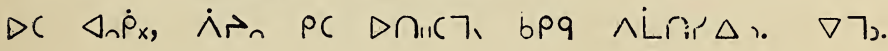


28

V.

BURIAL SERVICE.

PC $\left.j<C_{x} \quad \nabla e_{11} \Delta \sigma\right)_{x}$.

$\left(\Delta ৬ \Gamma_{\|} \nabla \Delta \cdot \neg \sigma_{0} \rho C \Delta U \cdot 0:-\right)$

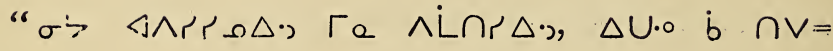

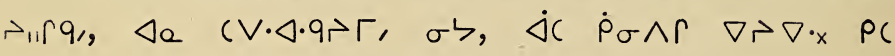

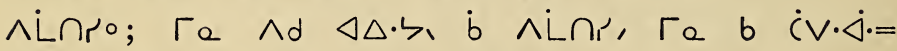

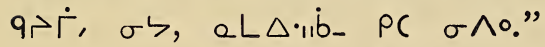

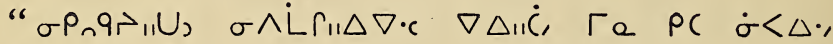

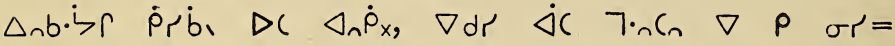

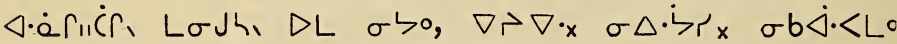

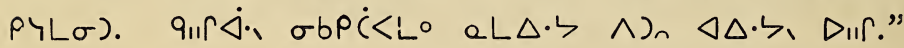

"a a qb: $p p \wedge_{11}\left(b \cdot \dot{C}_{2}{ }^{\circ} D C \quad \Delta_{n} \dot{p}_{x}, \nabla d r \quad q_{11} \Gamma_{a} D<b\right)$

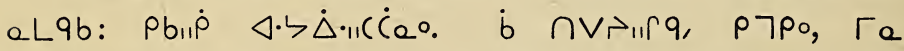

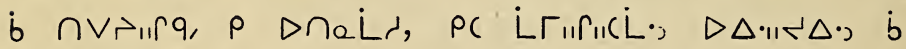

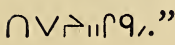

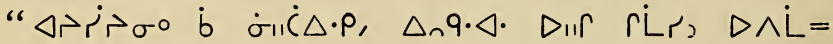

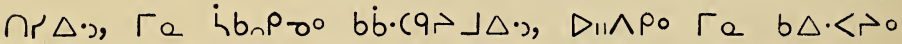

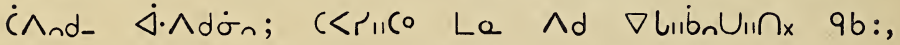

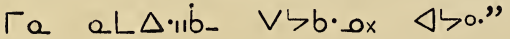

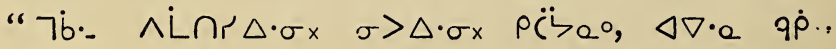


29

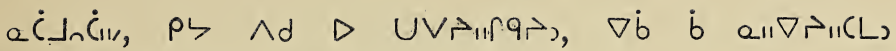
oLín $\triangle \cdot \sigma \dot{e} e$ DII ?"

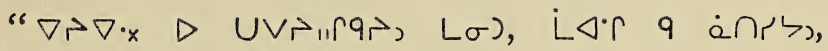

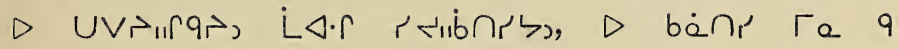

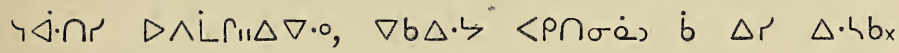
bpq $\sigma>\Delta \cdot{ }^{\prime}$ "

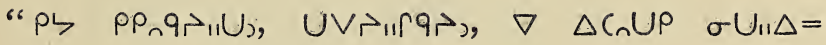

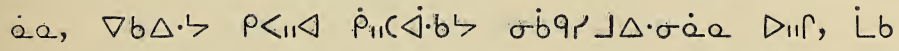

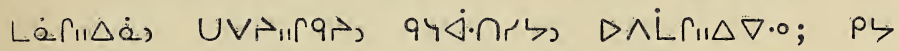

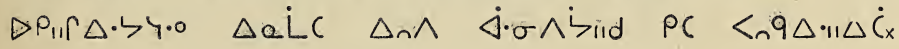
aco $\Delta r$.

(

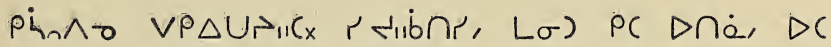

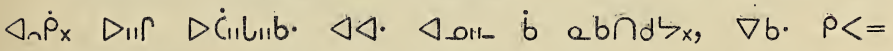

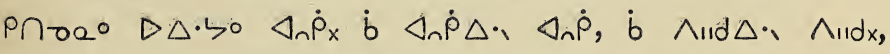

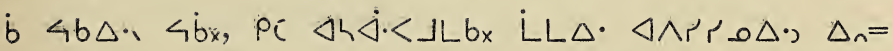

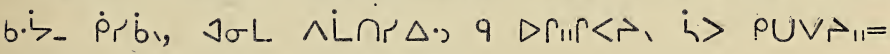

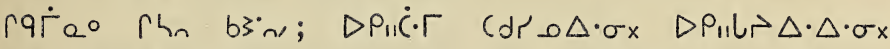

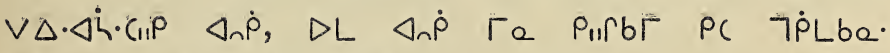

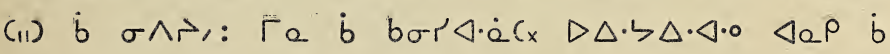

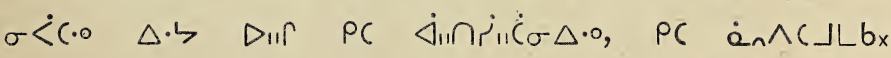

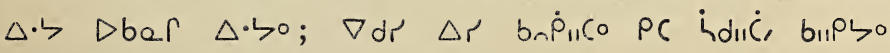
96: $q_{11}<\dot{1 .}$

$\left(\nabla d d^{\prime} \nabla b \cdot \rho C \Delta \dot{C} \cdot \sigma \Delta \cdot O:-\right)$

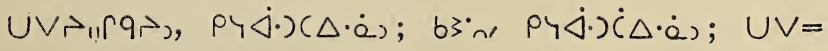

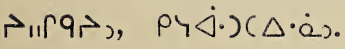


30

$\Delta ৬ \Gamma_{\|} \triangleleft \Delta \cdot \jmath$

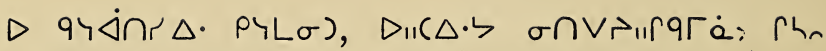

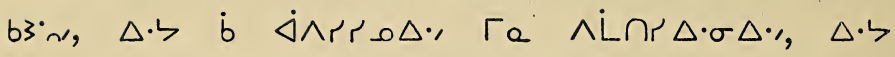

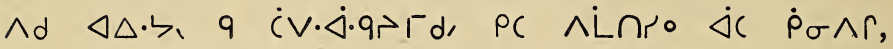

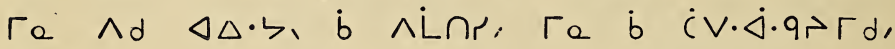

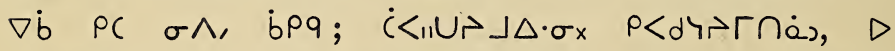

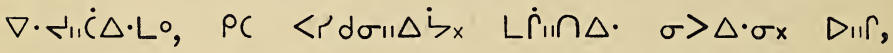

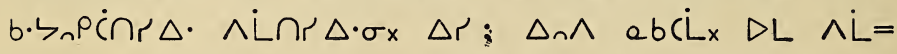

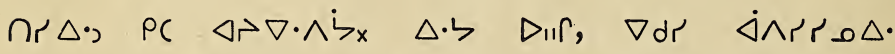

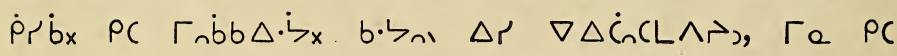

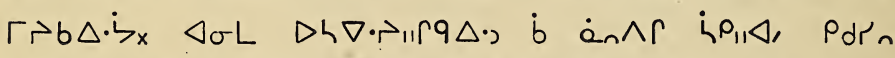

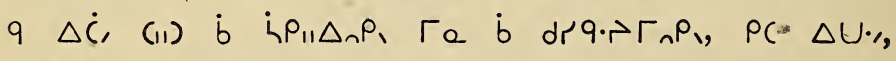

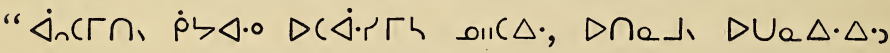
b $\triangleleft \cdot \nabla \cdot \dot{r}_{n}\left(\dot{L} b \Delta \cdot S_{1} \Delta_{n} \wedge\right)$ b $\Delta_{n} \dot{\rho} \Delta \cdot . . "$

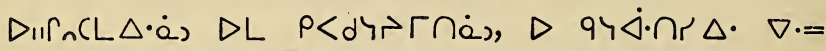

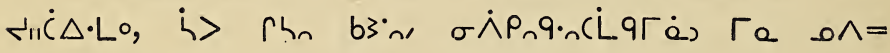
$\dot{L}_{11} \Delta \nabla \cdot \Gamma \dot{a}_{3}, \quad \nabla \eta_{\text {. }}$.

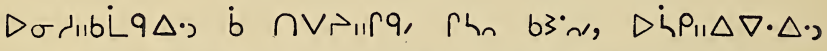

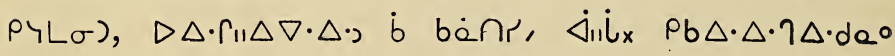

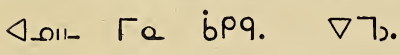


31

FORMS OF PRAYER FOR DIVINE SERVICE.

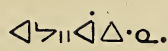

(i.)

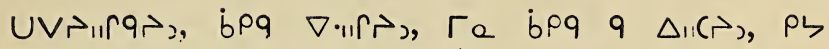

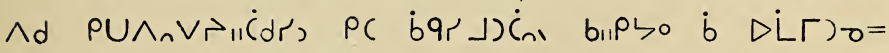

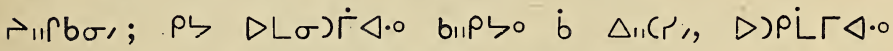

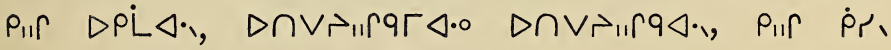

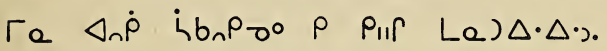

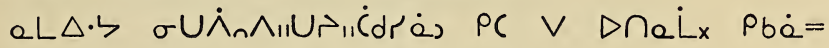

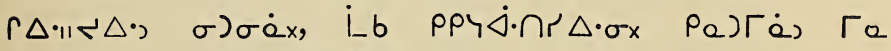
$(\Delta \cdot a\lrcorner \Delta \cdot \dot{a})$ Pr $V \dot{\alpha} \cap \dot{C} x$.

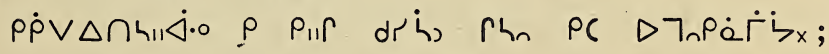

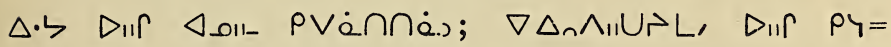

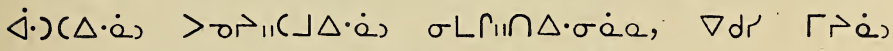

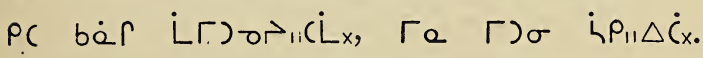

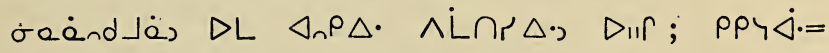

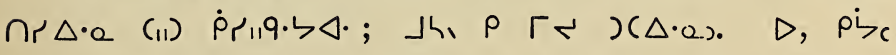

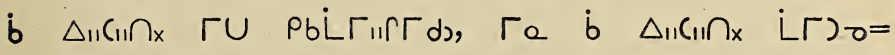
$r_{11}(b)$ Pba)ads; $\left.\nabla d r \quad \dot{b} \Delta_{11}(r\rangle_{x} \quad \sigma b \Delta \cdot<r d n \cup a\right)$

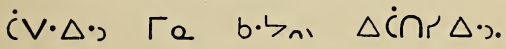

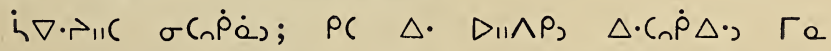

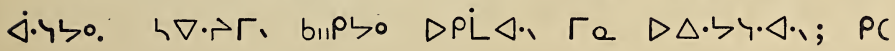


32

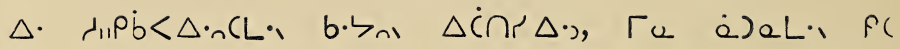
$r<>r$, bाए

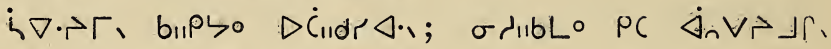

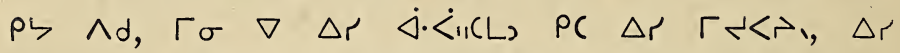

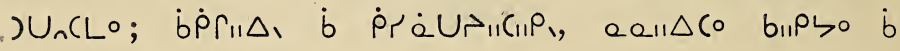
$\triangle \cdot\left\llcorner 9 \nabla_{\| C}\right\lrcorner\left\llcorner b_{x} \Gamma U\right.$.

$\left.\left.\left.\Gamma r \nabla \cdot \nabla \cap b \Gamma, \rho C \Delta \cdot D_{i 1} \wedge \dot{P} L b\right) \quad \rho \Gamma \dot{\triangleleft} \cdot \Gamma\right\lrcorner \Delta \cdot\right), \quad \nabla d r$

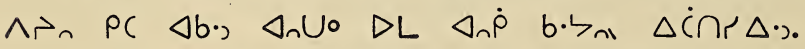

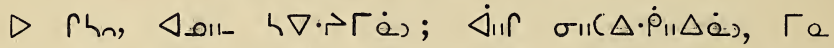

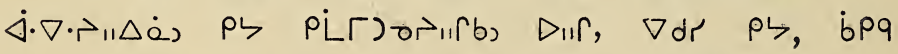
Pbilirrinä, $\Delta \cdot ל$ Dilr rin b3ंn. $\nabla 7$ ).

(2.)

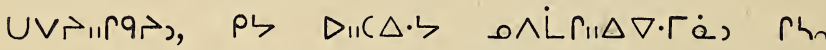

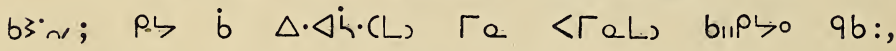

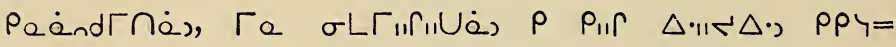

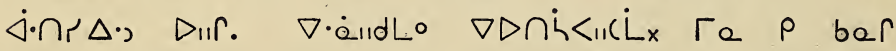

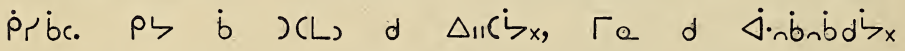

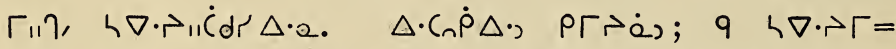

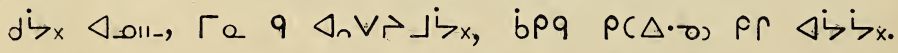

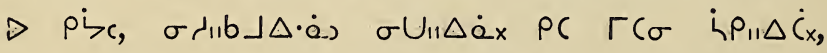

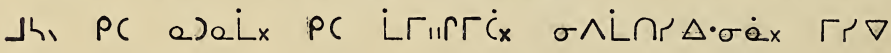

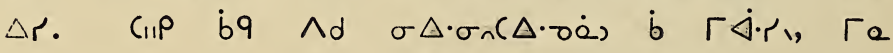

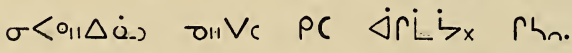

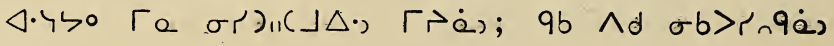


33

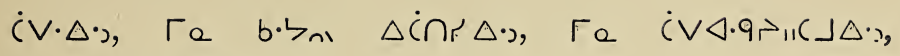
Te $\quad\left\langle p_{11} \dot{\Delta} \nabla \cdot \Delta \cdot \sigma_{0}\right.$

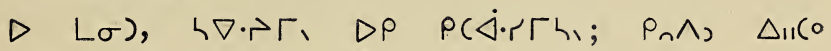

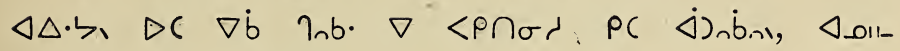
$\triangle r(11 \nabla) \quad P C \quad V \dot{a} \cap_{n}$.

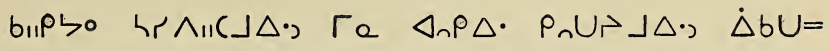

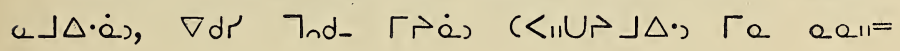
$\triangle{ }_{11}(\lrcorner \Delta \cdot{ }^{\prime} \cdot$

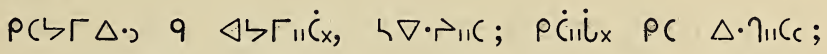

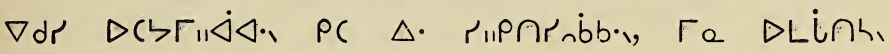
PC $\triangle \cdot$ dnde $\triangleleft \cdot 1 ; \quad \nabla d r$ bוp

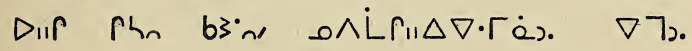

(3.)

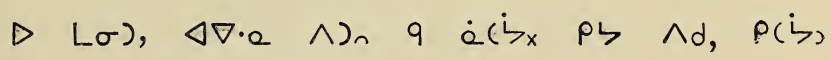
ALกR $\triangle \cdot \Delta \zeta \Gamma \Delta \cdot a$.

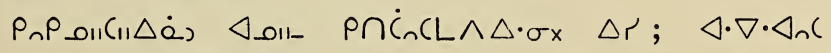

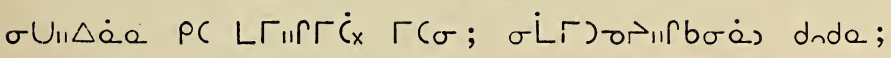

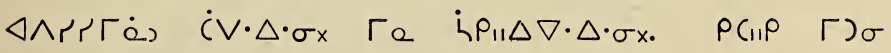
L $P_{11} \Delta \dot{C}_{x}$ Ph $\left.\left.\sigma L \sigma\right) \Gamma \dot{a}\right) \Gamma e$ rhn $\left.\circ \wedge \dot{L} \Gamma_{11} \Delta \nabla \cdot \Gamma \dot{Q}\right), \Delta r$

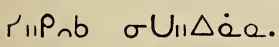

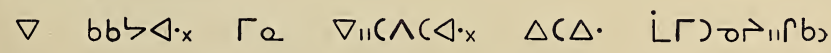

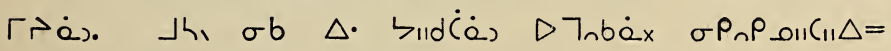
$\nabla \cdot \Gamma \dot{a}$, rhn.

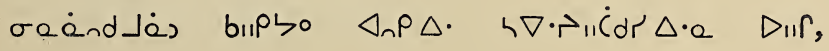


34

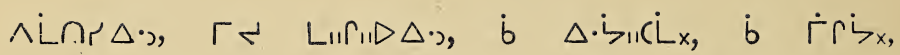

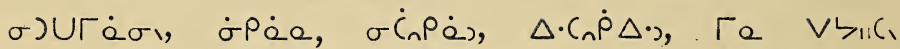

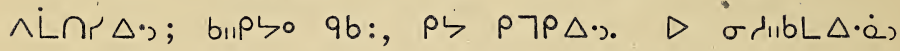

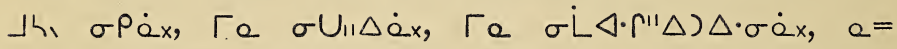

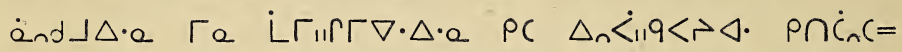

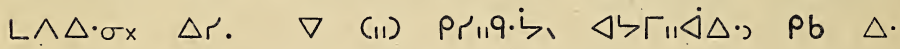

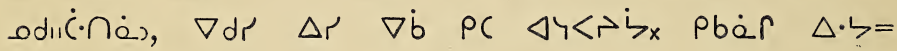
$\lambda \nabla \cdot \Delta \cdot \sigma x \quad D_{11}$.

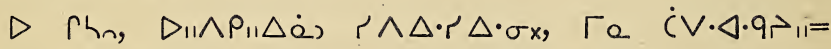

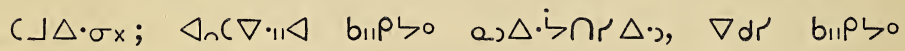
b $D) \cup \Gamma_{11} \Delta \nabla \cdot, \rho C \triangle \cdot D_{11} \wedge \rho, \sigma U_{11} \Delta \dot{\alpha}$.

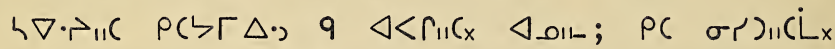
$\left.\left.\Gamma a, \quad \nabla \Gamma r \Delta \cdot \sigma \dot{C} \cdot \circ \quad \sigma \dot{C}_{11} \dot{L}_{11} \dot{a}_{2}, \quad \Delta r \quad \sigma d_{1} b\right\lrcorner \Delta \cdot \dot{a}\right)$.

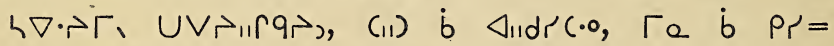

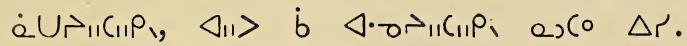

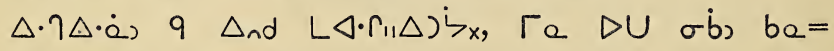

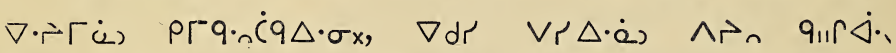

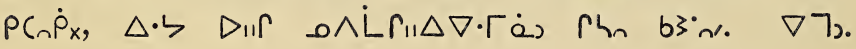


35

FORMS OF PRAYER IN CASES OF SICKNESS.

(1) Prayer for the. Sick of Riper Years.

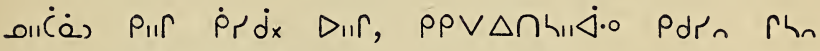

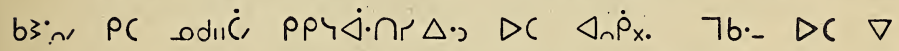

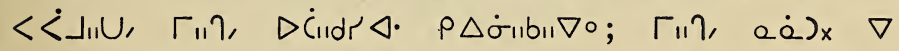

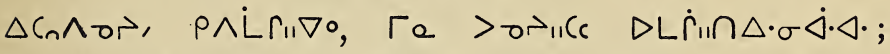

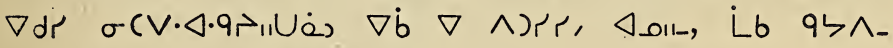

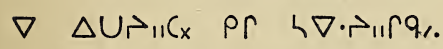

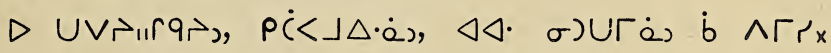

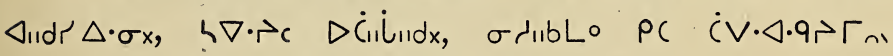

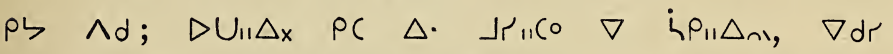

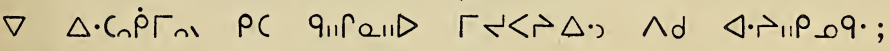

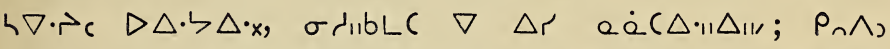

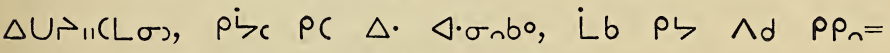

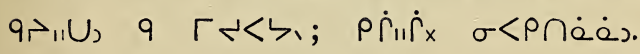

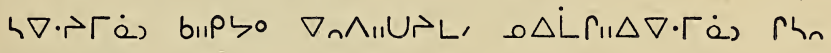
b3๋ ^. $\nabla 7$.

(2) Prayer for Sick Children.

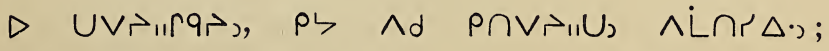

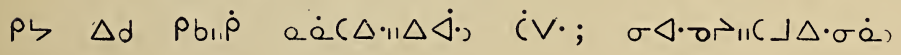


36

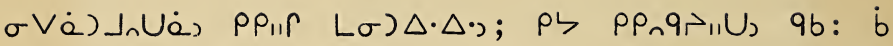

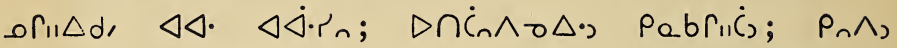

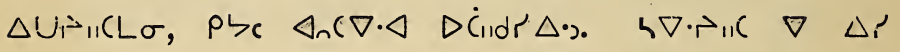

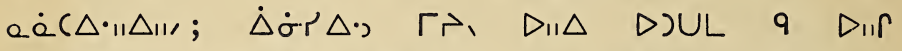

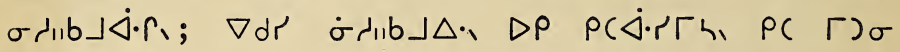

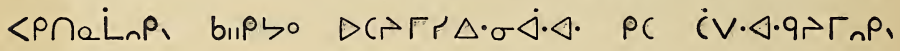
$\nabla \dot{b} \quad \dot{a}_{2} c_{0} \quad \Delta r \quad P C_{11} \dot{\rho}<C_{11} \triangleleft_{11} P_{1}$.

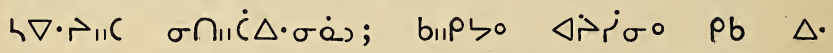

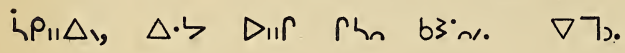





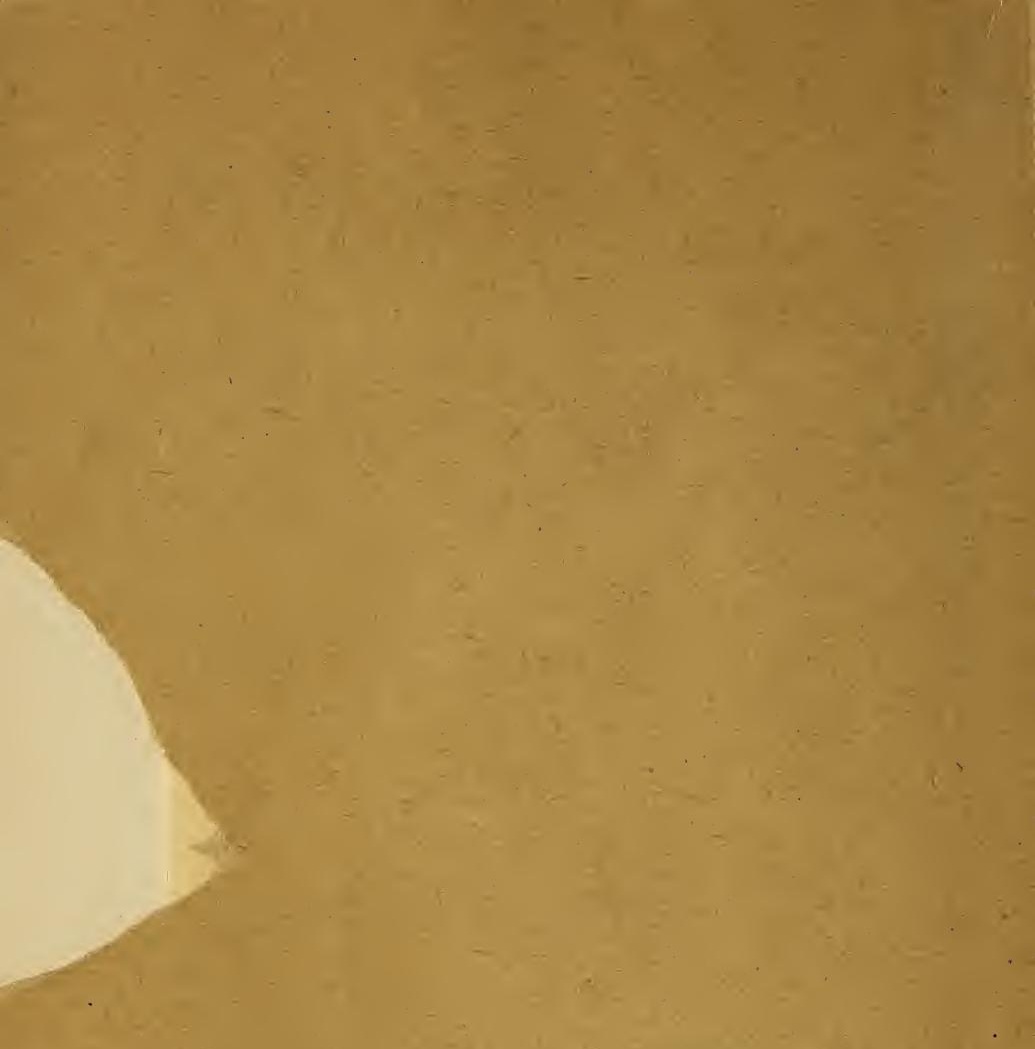

(1)

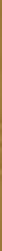

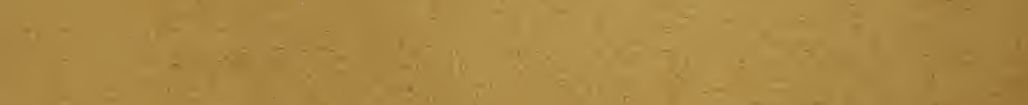

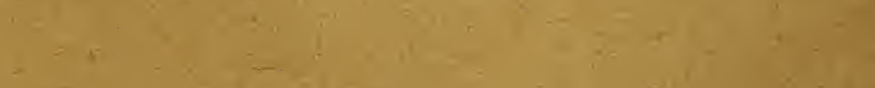

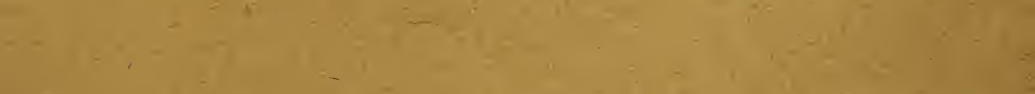

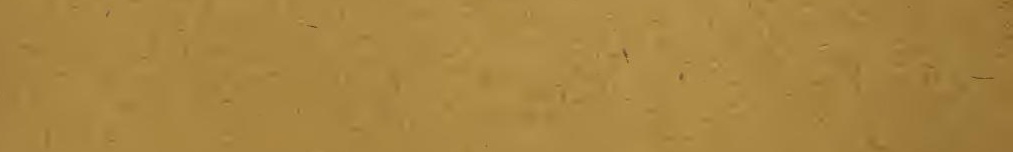
$=$ 4 